\title{
SPACE OF CHORD DIAGRAMS ON SPHERICAL CURVES
}

\author{
NOBORU ITO
}

\begin{abstract}
In this paper, we give a definition of $\mathbb{Z}$-valued functions from the ambient isotopy classes of spherical/plane curves derived from chord diagrams, denoted by $\sum_{i} \alpha_{i} x_{i}$. Then, we introduce certain elements of the free $\mathbb{Z}$-module generated by the chord diagrams with at most $l$ chords, called relators of Type (I) ((SII), (WII), (SIII), or (WIII), resp.), and introduce another function $\sum_{i} \alpha_{i} \tilde{x}_{i}$ derived from $\sum_{i} \alpha_{i} x_{i}$. The main result (Theorem 1 shows that if $\sum_{i} \alpha_{i} \tilde{x}_{i}$ vanishes for the relators of Type (I) ((SII), (WII), (SIII), or (WIII), resp.), then $\sum_{i} \alpha_{i} x_{i}$ is invariant under the Reidemeister move of type RI (strong RII, weak RII, strong RIII, or weak RIII, resp.) that is defined in [4.
\end{abstract}

\section{INTRODUCTION}

A spherical curve (plane curve, resp.) is the image of a generic immersion of a circle into a 2-sphere (plane, resp.). In this paper, we study certain equivalence classes of spherical/plane curves. Any two spherical/plane curves can be transformed into each other by a finite sequence of Reidemeister moves, each of which is either one of types RI, RII, or RIII that is a replacement of a part of the curve as Figure 1. These moves are obtained from Reidemeister moves of types $\Omega_{1}, \Omega_{2}$,

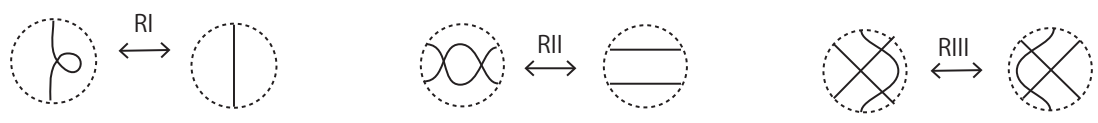

Figure 1. Reidemeister moves: types RI, RII, and RIII.

and $\Omega_{3}$ on knot diagrams by ignoring over/under information. It is well-known that two knot diagrams represent the same knot if and only if one knot diagram is transformed into the other knot diagram by a finite sequence of Reidemeister moves of types $\Omega_{1}, \Omega_{2}$, and $\Omega_{3}$.

In 1990, Vassiliev 9] defined an infinite sequence of knot invariants that are at least as powerful as all of the quantum group invariants of knots. After that some researchers gave alternative definitions of Vassiliev invariants (see 11). In 1994, Polyak and Viro 8 introduced a formulation of Vassiliev invariants using chord diagrams, and presented explicit formulas for some Vassiliev invariants. Goussarov [2] proved that the formulation of Polyak-Viro provides all Vassiliev invariants of knots. In 2001, Östlund further developed the theory of Polyak and Viro by using singularity theory of plane curves, and obtained the following result [7, Theorem 6]: if $v$ is a function on the set of knot diagrams that is invariant under plane isotopy, $\Omega_{1}$, and $\Omega_{3}$ and is so-called Vassiliev-type, then $v$ is invariant under $\Omega_{2}$. Motivated

Key words and phrases. spherical curve; plane curve; chord diagram; Reidemeister move.

MSC2010: Primary: 57R42, Secondary: 57M99. 
by this result, Östlund [7] posed the following question: can every plane curve be transformed into the simple closed curve by a finite sequence of Reidemeister moves of types RI and RIII?

In 2008, Hagge and Yazinski [3] showed that the answer to this question is negative, i.e., they showed that the equivalence classes under RI and RIII are nontrivial. In fact, any finite sequence of Reidemeister moves that transforms the plane curve depicted in Figure 2 into the simple closed curve contains a Reidemeister move of type RII. However, to the best of the author's knowledge, the equivalence class

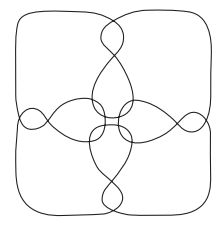

Figure 2. Example by Hagge and Yazinski [3].

containing the simple closed curve under RI and RIII has not been determined yet. In general, none of the equivalence classes under RI and RIII have been determined yet.

Viro [10] suggested the idea that type RIII is decomposed into the following two types: suppose that a plane curve $P_{1}$ is transformed into $P_{2}$ by a single RIII. In this paper, we apply the terminology to spherical curves. Note that in RIII in Figure 1 . a triangle is observed in each of the disks. We say that spherical/plane curves $P_{1}$ and $P_{2}$ are related by a strong $R I I I$ if the orientations on the edges of the triangle induced by an orientation of the spherical/plane curve are coherent. If $P_{1}$ and $P_{2}$ are not related by a strong RIII, then we say that $P_{1}$ and $P_{2}$ are related by a weak RIII. Analogously, for type RII, we define strong RII and weak RII as follows. Suppose that $P_{1}$ is transformed into $P_{2}$ by a single RII. We say that $P_{1}$ and $P_{2}$ are related by a strong $R I I$ if the orientations on the edges of the triangle induced by an orientation of the spherical/plane curve are coherent. If $P_{1}$ and $P_{2}$ are not related by a strong RII, then we say that $P_{1}$ and $P_{2}$ are related by a weak $R$ II. See Figure 3 .

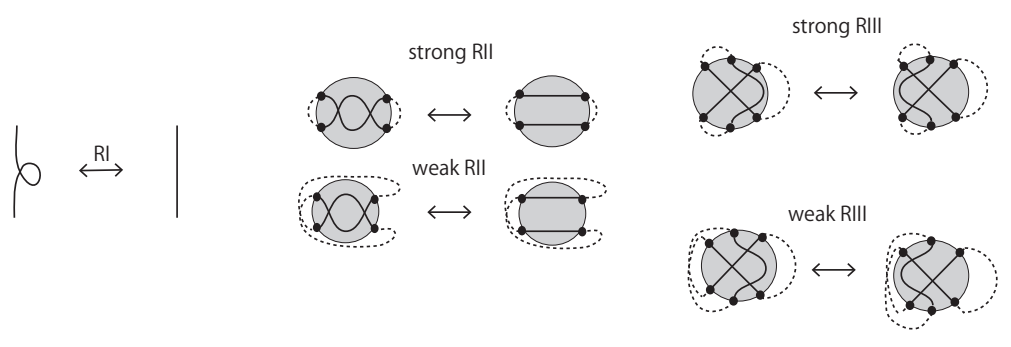

FiguRE 3. RI, strong RII, weak RII, strong RIII, and weak RIII.

From the viewpoint of Viro, the following problems arise from Östlund's question above: which spherical curve is transformed into the simple closed curve by a finite sequence of Reidemeister moves of types RI and strong RIII (types RI and weak RIII, resp.)? First, in 2013, a necessary and sufficient condition that a spherical curve 
and the simple closed curve are related by a finite sequence of Reidemeister moves of types RI and weak RIII was given [4. Second, in 2015, a necessary and sufficient condition that a spherical curve and the simple closed curve are related by a finite sequence of Reidemeister moves of types RI and strong RIII was given [6]. After that, in 2015, different necessary and sufficient conditions using invariants were given [5]. More concretely, for a chord diagram corresponding to a spherical curve, by counting the number of sub-chord diagrams of certain types, integer-valued

functions $\lambda, H$, and $\widetilde{X}$ were defined, and the following was shown: a spherical curve $P$ and the simple closed curve are related by a finite sequence of Reidemeister moves of types RI and strong RIII (types RI and weak RIII, resp.) if and only if $H(P)=0$ and $\lambda(P)=0(\widetilde{X}(P)=0$, resp. $)[5]$.

In this paper, we further develop the idea of counting sub-chord diagrams, which leads to functions of all equivalence classes of spherical/plane curves generated by all possible combinations of Reidemeister moves of types RI, strong RII, weak RII, strong RIII, and weak RIII. In [8], for a chord diagram $A$, and a chord diagram $G$ obtained from a knot diagram, Polyak and Viro define an integer $\langle A, G\rangle$. They further consider a linear combination of $\langle A, \cdot\rangle$, that is denoted by $\left\langle\sum_{i} r_{i} A_{i}, \cdot\right\rangle$, which is a function on knot diagrams, and show that this type of the function induces a non-trivial Vassiliev knot invariant. We will mimic their idea to define a function, denoted by $\sum \alpha_{i} x_{i}$, where $x_{i}$ denotes a chord diagram. Roughly speaking, $x_{i}(P)$ is obtained from $\langle A, G\rangle$ by ignoring "signs and arrows", and this means that we ignore the over/under information of crossings and orientations of knot diagrams. In Section 2, we introduce certain elements of the free $\mathbb{Z}$-module $\mathbb{Z}\left[G_{\leq l}\right]$ generated by the chord diagrams with at most $l$ chords, called relators of Type (I) ((SII), (WII), (SIII), or (WIII), resp.), and introduce another function $\sum_{i} \alpha_{i} \tilde{x}_{i}$ derived from $\sum_{i} \alpha_{i} x_{i}$. The main result (Theorem 1) shows that if $\sum_{i} \alpha_{i} \tilde{x}_{i}$ vanishes for the relators of Type (I) ((SII), (WII), (SIII), or (WII), resp.), then $\sum_{i} \alpha_{i} x_{i}$ is invariant under RI (strong RII, weak RII, strong RIII, or weak RIII, resp.). In Section 4 we show that Theorem 1 produces a new function that is invariant under RI and strong RIII and is able to distinguish some pairs which the function $\lambda$ of [5] cannot distinguish.

\section{Preliminaries AND MAin Results}

\subsection{Definitions and notations.}

Definition 1 (Gauss word). A word $w$ of length $n$ is a map from $\hat{n}=\{1,2,3, \ldots, n\}$ to $\mathbb{N}$. This word is represented by $w(1) w(2) w(3) \cdots w(n)$. For a word $w: \hat{n} \rightarrow \mathbb{N}$, we call each element of $w(\hat{n})$ a letter. A word $u$ of length $q$ is a sub-word of $w$ if there is an integer $p(q \leq p \leq n)$ such that $u(j)=w(n-p+j)(1 \leq j \leq q)$. A Gauss word of length $2 n$ is a word $w$ of length $2 n$ where each letter in $w(2 n)$ appears exactly twice in $w(1) w(2) w(3) \cdots w(2 n)$. Let cyc and rev be maps $\hat{2 n} \rightarrow 2 \hat{n}$ such that $\operatorname{cyc}(p) \equiv p+1(\bmod 2 n)$ and $\operatorname{rev}(p) \equiv-p+1(\bmod 2 n)$. Two Gauss words, $v$ and $w$, of length $2 n$ are isomorphic if there exists a bijection $f: v(\hat{2 n}) \rightarrow w(\hat{2 n})$ satisfying the following: there exists $t \in \mathbb{Z}$ such that $w \circ(\mathrm{cyc})^{t} \circ(\mathrm{rev})^{\epsilon}=f \circ v$ $(\epsilon=0$ or 1$)$. The isomorphisms give an equivalence relation on the Gauss words. For a Gauss word $v$ of length $2 n,[v]$ denotes the equivalence class containing $v$. A Gauss word $v^{\prime}$ is a sub-Gauss word of the Gauss word $v$ if $v^{\prime}$ is obtained from $v$ by ignoring some letters of $v$. Then $\operatorname{Sub}(v)$ denotes the set of sub-Gauss words of $v$. 
Definition 2 (chord diagram). Each configuration of $n$ pair(s) of points on a circle up to ambient isotopy and reflection of the circle is called a chord diagram. The integer $n$ is called the length of the chord diagram. Traditionally, two points of each pair are connected by a straight arc, called a chord.

We note that the equivalence classes of Gauss words of length $2 n$ have one to one correspondence with the chord diagrams, each of which has $n$ chords as in Figure 4 . In the rest of this paper, we identify these four expressions in Figure 4, and freely

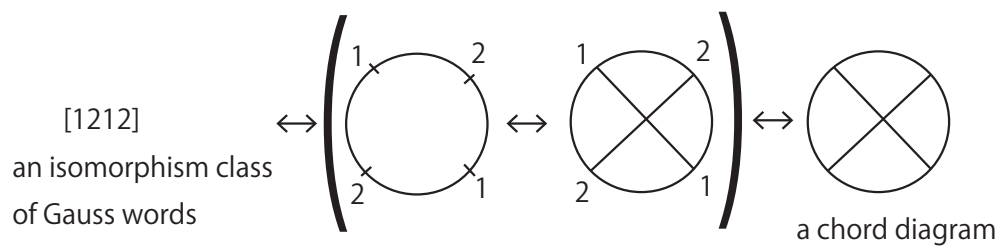

FiguRE 4. Four expressions.

use either one of them depending on the situation.

Notation $1\left(G_{\leq d}, n_{d}, G_{b, d}\right)$. Let $G_{<\infty}$ be the set of chord diagrams, that is, the set of isomorphism classes of Gauss words. It is clear that $G_{<\infty}$ consists of countably many elements. Hence, there exists a bijection between $G_{<\infty}$ and $\left\{x_{i}\right\}_{i \in \mathbb{N}}$, where $x_{i}$ is a variable. Take and fix a bijection $f: G_{<\infty} \rightarrow\left\{x_{i}\right\}_{i \in \mathbb{N}}$ satisfying: the number of chords of $f^{-1}\left(x_{i}\right)$ is less than or equal to that of $f^{-1}\left(x_{j}\right)$ if and only if $i \leq j(i, j \in \mathbb{N})$. For each positive integer $d$, let $G_{\leq d}$ be the set of chord diagrams consisting of at most $d$ chords and let $n_{d}=\left|G_{\leq d}\right|$. Then, it is clear that $\left.f\right|_{G_{<d}}$ is a bijection from $G_{\leq d}$ to $\left\{x_{1}, x_{2}, \ldots, x_{n_{d}}\right\}$. Further, for each pair of integers $b$ and $d(2 \leq b \leq d)$, let $G_{b, d}=G_{\leq d} \backslash G_{\leq b-1}$. Then, $\left.f\right|_{G_{b, d}}$ is a bijection $G_{b, d} \rightarrow$ $\left\{x_{n_{b-1}+1}, x_{n_{b-1}+2}, \ldots, x_{n_{d}}\right\}$.

In the rest of this paper, we use the notations in Notation 1 unless otherwise denoted, and we freely use this identification between $G_{<\infty}$ and $\left\{x_{i}\right\}_{i \in \mathbb{N}}$.

Definition 3 (a chord diagram $C D_{P}$ of a spherical/plane curve $P$ ). Let $P$ be a spherical/plane curve, i.e. there is a generic immersion $g: S^{1} \rightarrow S^{2}$ or $\mathbb{R}^{2}$ such that $g\left(S^{1}\right)=P$. We define a chord diagram of $P$ (e.g., Figure 5 ) as follows: let $k$ be the number of the double points of $P$, and $m_{1}, m_{2}, \ldots, m_{k}$ mutually distinct positive integers. Fix a base point, which is not a double point on $P$, and choose an orientation of $P$. Starting from the base point, proceed along $P$ according to the orientation of $P$. We assign $m_{1}$ to the first double point that we encounter. Then we assign $m_{2}$ to the next double point that we encounter provided it is not the first double point. Suppose that we have already assigned $m_{1}, m_{2}, \ldots, m_{p}$. Then we assign $m_{p+1}$ to the next double point that we encounter if it has not been assigned yet. Following the same procedure, we finally label all the double points. Note that $g^{-1}$ (double point assigned $m_{i}$ ) consists of two points on $S^{1}$ and we shall assign $m_{i}$ to them. The chord diagram represented by $g^{-1}$ (double point assigned $m_{1}$ ), $g^{-1}$ (double point assigned $\left.m_{2}\right), \ldots, g^{-1}$ (double point assigned $\left.m_{k}\right)$ on $S^{1}$ is denoted by $C D_{P}$ and is called a chord diagram of the spherical/plane curve $P$. 


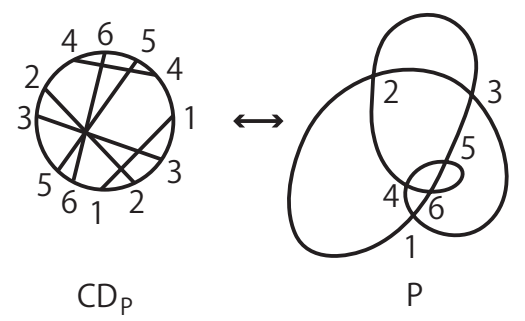

Figure 5. A chord diagram $C D_{P}$ of a spherical/plane curve $P$.

Recall that $C D_{P}$ gives an equivalence class of Gauss words, say $\left[v_{P}\right]$. Then, by the definition of the equivalence relation, it is easy to see that the map $P \mapsto\left[v_{P}\right]$ is well-defined.

Notation $2(x(C D))$. Let $x \in\left\{x_{i}\right\}_{i \in \mathbb{N}}$ (hence, $x$ corresponds to a chord diagram). For a given chord diagram $C D$, fix a Gauss word $G$ representing $C D$. Let $\operatorname{Sub}_{x}(G)$ $=\{H \mid H \in \operatorname{Sub}(G),[H]=x\}$. The cardinality of this subset is denoted by $x(G)$, i.e., $x(G)=\left|\operatorname{Sub}_{x}(G)\right|$. Let $G^{\prime}$ be another Gauss word representing $C D$. By the definition of the isomorphism of Gauss words, it is easy to see $x\left(G^{\prime}\right)=x(G)$. Hence, we shall denote this number by $x(C D)$. If $C D$ is a chord diagram of a spherical/plane curve $P$, then $x(C D)$ can be denoted by $x(P)$.

Since each equivalence class of Gauss words is identified with a chord diagram, we can calculate the number $x(C D)$ using geometric observations. We explain this philosophy in the next example.

Example 1. We consider the chord diagram $C D$ in Figure 6 (Note that $C D=$ $C D_{P}$ in Figure 5). Then we label the chords of $C D$ by $\alpha_{i}(1 \leq i \leq 6)$ as in Figure 6. Consider the subset of the power set of $\left\{\alpha_{1}, \alpha_{2}, \ldots, \alpha_{6}\right\}$, each element of which represents a chord diagram isomorphic to $\otimes$. It is elementary to see that this subset consists of ten elements, those are, $\left\{\alpha_{1}, \alpha_{2}\right\},\left\{\alpha_{1}, \alpha_{3}\right\},\left\{\alpha_{1}, \alpha_{4}\right\},\left\{\alpha_{2}, \alpha_{3}\right\}$, $\left\{\alpha_{2}, \alpha_{4}\right\},\left\{\alpha_{3}, \alpha_{4}\right\},\left\{\alpha_{1}, \alpha_{5}\right\},\left\{\alpha_{2}, \alpha_{5}\right\},\left\{\alpha_{3}, \alpha_{6}\right\}$, and $\left\{\alpha_{4}, \alpha_{6}\right\}$, and that fact shows that $\otimes(C D)=10$. Similarly, we have $\boxplus(C D)=6$ and $\bigoplus(C D)=8$.

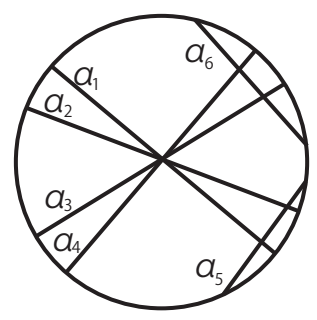

Figure $6 . C D$.

Let $C D$ be a chord diagram consisting of chords $\alpha_{1}, \alpha_{2}, \ldots, \alpha_{k}$. Then the chord diagram consisting of a subset of $\left\{\alpha_{1}, \alpha_{2}, \ldots, \alpha_{k}\right\}$ is called a sub-chord diagram of $C D$. 
Definition $4(\tilde{x}(z), \tilde{x}([z]))$. Let $x$ be a chord diagram. We define the function $\tilde{x}$ from the set of Gauss words to $\{0,1\}$ by

$$
\tilde{x}(F)= \begin{cases}1 & {[F]=x} \\ 0 & {[F] \neq x .}\end{cases}
$$

By definition, it is easy to see $\tilde{x}\left(F_{1}\right)=\tilde{x}\left(F_{2}\right)$ for each pair $F_{1}$ and $F_{2}$ with $\left[F_{1}\right]=\left[F_{2}\right]$. Hence, we shall denote this number by $\tilde{x}\left(\left[F_{1}\right]\right)$. If $[F]$ corresponds to a chord diagram of a spherical/plane curve $P$, then $\tilde{x}([F])$ is denoted by $\tilde{x}(P)$. Further, let $\mathbb{Z}\left[G_{\leq l}\right]$ be the free $\mathbb{Z}$-module generated by the elements of $G_{\leq l}$, where $l$ is sufficiently large. We linearly extend $\tilde{x}$ to a function from $\mathbb{Z}\left[G_{\leq l}\right]$ to $\mathbb{Z}$. It is clear that for any Gauss word with $[G]=C D$,

$$
x(C D)=\sum_{z \in \operatorname{Sub}(G)} \tilde{x}(z) .
$$

2.2. Relators and Reidemeister moves. Let $\mathbb{Z}\left[G_{\leq l}\right]$ be the free $\mathbb{Z}$-module defined in Subsection 2.1. In this subsection, first we define the elements of $\mathbb{Z}\left[G_{<l}\right]$ called relators of types (I), (SII), (WII), (SIII), and (WIII).

Definition 5 (Relators, cf. Figure 7). - Type (I). An element $r$ of $\mathbb{Z}\left[G_{\leq l}\right]$ is called a Type (I) relator if there exist a Gauss word $S$ and a letter $i$ not in $S$ such that $r=[$ Sii $]$.

- Type (SII). An element $r$ of $\mathbb{Z}\left[G_{\leq l}\right]$ is called a Type (SII) relator if there exist a Gauss word $S T$ and letters $i$ and $j$ not in $S T$ such that $r=[S i j T j i]$ $+[S i T i]+[S j T j]$.

- Type (WII). An element $r$ of $\mathbb{Z}\left[G_{\leq l}\right]$ is called a Type (WII) relator if there exists a Gauss word $S T$ and letters $i$ and $j$ not in $S T$ such that $r=[S i j T i j]$ $+[S i T i]+[S j T j]$.

- Type (SIII). An element $r$ of $\mathbb{Z}\left[G_{\leq l}\right]$ is called a Type (SIII) relator if there exists a Gauss word $S T U$ and letters $i, j, k$ not in $S T U$ such that $r=$ $([$ SijTkiUjk $]+[$ SijTiUj] $]+[$ SiTkiUk $]+[$ SjTkUjk $])-([$ SjiTikUkj] $]+$ $[$ SjiTiUj] $+[$ SiTikUk] $+[$ SjTkUkj]).

- Type (WIII). An element $r$ of $\mathbb{Z}\left[G_{\leq l}\right]$ is called a Type (WIII) relator if there exists a Gauss word $S T U$ and letters $i, j, k$ not in $S T U$ such that $r=$ $([S i j T i k U j k]+[$ SijTiUj] $]+[$ SiTikUk] $]+[$ SjTkUjk] $]-([$ SjiTkiUkj] $]+$ $[S j i T i U j]+[S i T k i U k]+[S j T k U k j])$.

We note that in Definition 5 , each relator is induced by the corresponding Reidemeister move: Type (I) relator corresponding to RI, Type (SII) relator corresponding to strong RII, Type (WII) relator corresponding to weak RII, Type (SIII) relator corresponding to strong RIII, and Type (WIII) relator corresponding to weak RIII. For the precise statement of this note, we introduce the following setting.

We first introduce the following notation. Let $P$ and $P^{\prime}$ be two spherical/plane curves. If $P$ and $P^{\prime}$ are related by a single RI (strong RII, weak RII, strong RIII, or weak RIII resp.), then there are Gauss words $G$ and $G^{\prime}$ such that (by exchanging $P$ and $P^{\prime}$, if necessary), $G=S i i$ (SijTji, SijTij, SijTkiUjk, or SijTikUjk resp.) and $G^{\prime}=S\left(S T, S T, S j i T i k U k j\right.$, or $S j i T k i U k j$ resp.) such that $[G]=C D_{P}$ and $\left[G^{\prime}\right]=C D_{P^{\prime}}$ (Figure 8). The subset of $\operatorname{Sub}(G)$ such that each element has exactly $m$ letters of $i, j$, and $k$ is denoted by $\operatorname{Sub}^{(m)}(G)$. By definition,

$$
\operatorname{Sub}(G)=\operatorname{Sub}^{(0)}(G) \amalg \operatorname{Sub}^{(1)}(G) \amalg \operatorname{Sub}^{(2)}(G) \amalg \operatorname{Sub}^{(3)}(G) .
$$


( 1$)$

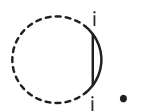

(SII)

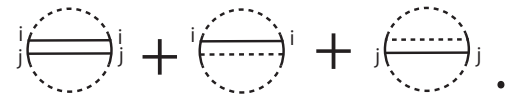

( WII )
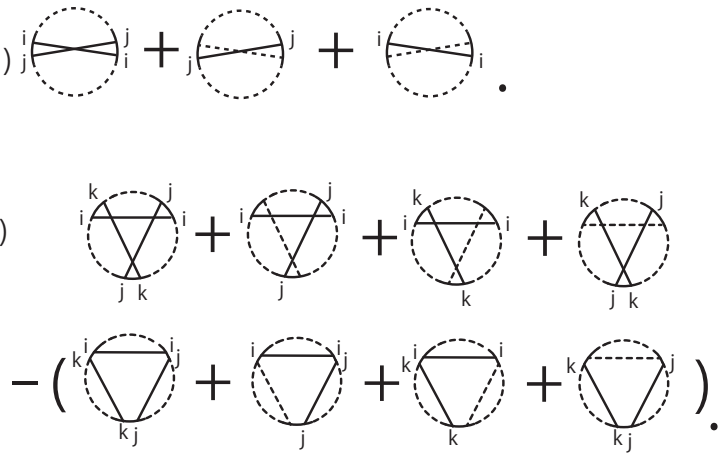

(WIII)
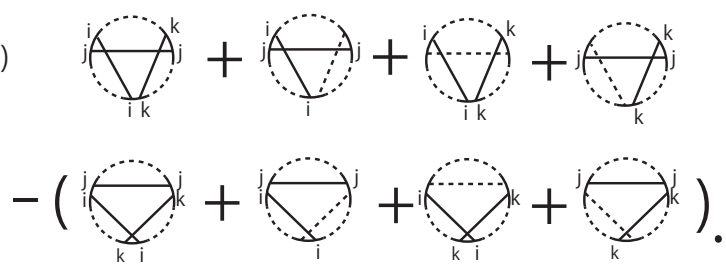

FIgURE 7. Relators.

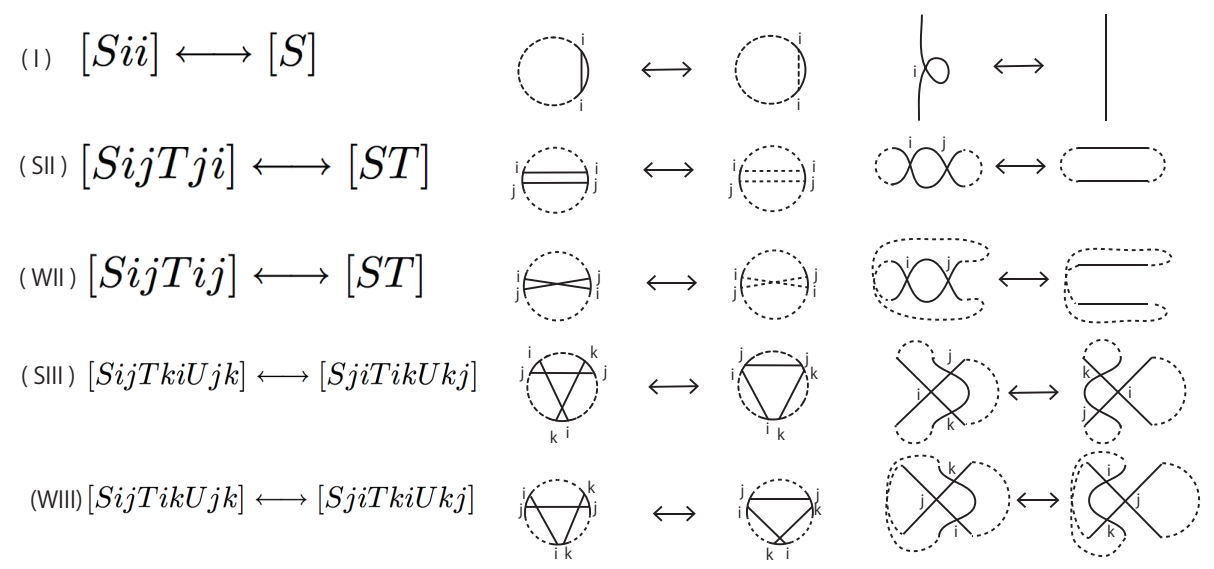

FiguRE 8. Reidemeister moves.

Similarly, for a chord diagram $x, \operatorname{Sub}_{x}^{(m)}(G)$ denotes the subset of $\operatorname{Sub}_{x}(G)$ consisting of elements, each of which has exactly $m$ letters of $i, j$, and $k$. Then,

$$
\operatorname{Sub}_{x}(G)=\operatorname{Sub}_{x}^{(0)}(G) \amalg \operatorname{Sub}_{x}^{(1)}(G) \amalg \operatorname{Sub}_{x}^{(2)}(G) \amalg \operatorname{Sub}_{x}^{(3)}(G) .
$$


Let $\mathcal{C}$ be the set of ambient isotopy classes of spherical/plane curves. Next, for each element $\sum_{i} \alpha_{i} x_{i} \in \mathbb{Z}\left[G_{\leq l}\right]$, we define a function $\mathcal{C} \rightarrow \mathbb{Z}$, also denoted by $\sum_{i} \alpha_{i} x_{i}$, and another function denoted by $\sum_{i} \alpha_{i} \tilde{x}_{i}$.

Definition $6\left(\sum_{i} \alpha_{i} x_{i}, \sum_{i} \alpha_{i} \tilde{x}_{i}\right)$. Let $b$ and $d(2 \leq b \leq d)$ be integers and $G_{\leq d}$, $G_{b, d},\left\{x_{i}\right\}_{i \in \mathbb{N}}$, and $\mathbb{Z}\left[G_{\leq l}\right]$ be as in Subsection 2.1. Recall that $n_{d}=\left|G_{\leq d}\right|$ and $G_{b, d}$ $=\left\{x_{i}\right\}_{n_{b-1}+1 \leq i \leq n_{d}}$ and each $x_{i}$ represents the chord diagram $f^{-1}\left(x_{i}\right)$. For each element

$$
\sum_{n_{b-1}+1 \leq i \leq n_{d}} \alpha_{i} x_{i} \in \mathbb{Z}\left[G_{\leq l}\right],
$$

we define an integer-valued function $\mathcal{C} \rightarrow \mathbb{Z}$, also denoted by $\sum_{n_{b-1}+1 \leq i \leq n_{d}} \alpha_{i} x_{i}$, by

$$
P \mapsto \sum_{n_{b-1}+1 \leq i \leq n_{d}} \alpha_{i} x_{i}(P),
$$

where $x_{i}(P)$ is the integer introduced in Notation 2 .

Analogously, for each $\sum_{n_{b-1}+1 \leq i \leq n_{d}} \alpha_{i} x_{i} \in \mathbb{Z}\left[G_{\leq l}\right]$, we define the function

$$
\sum_{n_{b-1}+1 \leq i \leq n_{d}} \alpha_{i} \tilde{x}_{i}: \mathbb{Z}\left[G_{\leq l}\right] \rightarrow \mathbb{Z}
$$

by

$$
\left(\sum_{n_{b-1}+1 \leq i \leq n_{d}} \alpha_{i} \tilde{x}_{i}\right)(P)=\sum_{n_{b-1}+1 \leq i \leq n_{d}} \alpha_{i} \tilde{x}_{i}(P),
$$

where $\tilde{x}_{i}(P)$ is the integer introduced in Definition 4.

Recall that if a spherical/plane curve $P$ and another spherical/plane curve $P^{\prime}$ are related by a single RI, then there exist a letter $i$ and a Gauss word $S$ such that $C D_{P}=[$ Sii $]$ and $C D_{P^{\prime}}=[S]$. Note that in this case in the decomposition $(2.2)$, $\operatorname{Sub}^{(2)}(G)=\emptyset$ and $\operatorname{Sub}^{(3)}(G)=\emptyset$, where $G=S i i$. Then, by 2.1) in Definition 4 and 2.2 ,

$$
\begin{aligned}
\sum_{n_{b-1}+1 \leq i \leq n_{d}} \alpha_{i} x_{i}(P) & =\sum_{n_{b-1}+1 \leq i \leq n_{d}} \alpha_{i}\left(\sum_{z \in \operatorname{Sub}(G)} \tilde{x}_{i}(z)\right) \\
& =\sum_{n_{b-1}+1 \leq i \leq n_{d}} \alpha_{i}\left(\sum_{z_{0} \in \operatorname{Sub}^{(0)}(G)} \tilde{x}_{i}\left(z_{0}\right)+\sum_{z_{1} \in \operatorname{Sub}^{(1)}(G)} \tilde{x}_{i}\left(z_{1}\right)\right) .
\end{aligned}
$$

Note that each element $z_{0} \in \operatorname{Sub}^{(0)}(G)$ is a sub-Gauss word of $S$. Then it is clear that $\operatorname{Sub}^{(1)}(G)=\left\{z_{0} i i \mid z_{0} \in \operatorname{Sub}^{(0)}(G)\right\}$. Hence,

$$
\begin{aligned}
\sum_{n_{b-1}+1 \leq i \leq n_{d}} \alpha_{i} x_{i}(P) & =\sum_{n_{b-1}+1 \leq i \leq n_{d}} \alpha_{i}\left(\sum_{z_{0} \in \operatorname{Sub}^{(0)}(G)} \tilde{x}_{i}\left(z_{0}\right)+\sum_{z_{0} \in \operatorname{Sub}^{(0)}(G)} \tilde{x}_{i}\left(z_{0} i i\right)\right) \\
& =\sum_{n_{b-1}+1 \leq i \leq n_{d}} \alpha_{i}\left(\sum_{z_{0} \in \operatorname{Sub}^{(0)}(G)} \tilde{x}_{i}\left(\left[z_{0}\right]\right)+\sum_{z_{0} \in \operatorname{Sub}^{(0)}(G)} \tilde{x}_{i}\left(\left[z_{0} i i\right]\right)\right) .
\end{aligned}
$$


On the other hand, since $\operatorname{Sub}\left(G^{\prime}\right)$ is identified with $\operatorname{Sub}^{(0)}(G)$,

$$
\begin{aligned}
\sum_{n_{b-1}+1 \leq i \leq n_{d}} \alpha_{i} x_{i}\left(P^{\prime}\right) & =\sum_{n_{b-1}+1 \leq i \leq n_{d}} \alpha_{i}\left(\sum_{z^{\prime} \in \operatorname{Sub}\left(G^{\prime}\right)} \tilde{x}_{i}\left(z^{\prime}\right)\right)(\because 2.1 p) \\
& =\sum_{n_{b-1}+1 \leq i \leq n_{d}} \alpha_{i}\left(\sum_{z_{0} \in \operatorname{Sub}^{(0)}(G)} \tilde{x}_{i}\left(z_{0}\right)\right) \\
& =\sum_{n_{b-1}+1 \leq i \leq n_{d}} \alpha_{i}\left(\sum_{z_{0} \in \operatorname{Sub}^{(0)}(G)} \tilde{x}_{i}\left(\left[z_{0}\right]\right)\right) .
\end{aligned}
$$

As a conclusion, the difference of the values is calculated as follows.

$$
\begin{aligned}
\sum_{n_{b-1}+1 \leq i \leq n_{d}} \alpha_{i} x_{i}(P) & -\sum_{n_{b-1}+1 \leq i \leq n_{d}} \alpha_{i} x_{i}\left(P^{\prime}\right) \\
& =\sum_{n_{b-1}+1 \leq i \leq n_{d}} \sum_{z_{0} \in \operatorname{Sub}^{(0)}(G)} \alpha_{i} \tilde{x}_{i}\left(\left[z_{0} i i\right]\right) .
\end{aligned}
$$

We note that this is a linear combination of the values of Type (I) relators via $\tilde{x}_{i}$.

For the cases of Type (SII), (WII), (SIII), or (WIII) relators, the arguments are slightly more complicated than that of Type (I) relator. We will explain them in the proof of Theorem 1 and thus, we omit them here.

Definition $7\left(R_{\epsilon_{1} \epsilon_{2} \epsilon_{3} \epsilon_{4} \epsilon_{5}}\right)$. For each $\left(\epsilon_{1}, \epsilon_{2}, \epsilon_{3}, \epsilon_{4}, \epsilon_{5}\right) \in\{0,1\}^{5}$, let $R_{\epsilon_{1} \epsilon_{2} \epsilon_{3} \epsilon_{4} \epsilon_{5}}=$ $\cup_{\epsilon_{i}=1} R_{i}\left(\subset \cup_{l \geq 1} \mathbb{Z}\left[G_{\leq l}\right]\right)$, where $R_{1}$ is the set of Type (I) relators, $R_{2}$ is the set of Type (SII) relators, $R_{3}$ is the set of Type (WII) relators, $R_{4}$ is the set of Type (SIII) relators, and $R_{5}$ is the set of Type (WII) relators.

For integers $b$ and $d(2 \leq b \leq d)$, let $O_{b, d}$ be the projection $\mathbb{Z}\left[G_{\leq l}\right] \rightarrow \mathbb{Z}\left[G_{b, d}\right]$. Here, note that $O_{b, d}$ is a linear map. By the definition, we immediately have:

Lemma 1. If $n_{b-1}+1 \leq i \leq n_{d}$, then for any $r \in \mathbb{Z}\left[G_{\leq l}\right]$,

$$
\tilde{x}_{i}(r)=\tilde{x}_{i}\left(O_{b, d}(r)\right) \text {. }
$$

Notation 3. Let $R_{\epsilon_{1} \epsilon_{2} \epsilon_{3} \epsilon_{4} \epsilon_{5}}(b, d)=O_{b, d}\left(R_{\epsilon_{1} \epsilon_{2} \epsilon_{3} \epsilon_{4} \epsilon_{5}}\right)$.

By using Lemma 1, we have the next proposition.

Proposition 1. For each pair of integers $b$ and $d(2 \leq b \leq d)$, let $\sum_{n_{b-1}+1 \leq i \leq n_{d}} \alpha_{i} \tilde{x}_{i}$ be a function as in Definition [6. For $\left(\epsilon_{1}, \epsilon_{2}, \epsilon_{3}, \epsilon_{4}, \epsilon_{5}\right) \in\{0,1\}^{5}$, let $R_{\epsilon_{1} \epsilon_{2} \epsilon_{3} \epsilon_{4} \epsilon_{5}}$ be the set as in Definition 7 . The following two statements are equivalent:

(1) $\sum_{n_{b-1}+1 \leq i \leq n_{d}} \alpha_{i} \tilde{x}_{i}(r)=0 \quad\left(\forall r \in R_{\epsilon_{1} \epsilon_{2} \epsilon_{3} \epsilon_{4} \epsilon_{5}}\right)$.

(2) $\sum_{n_{b-1}+1 \leq i \leq n_{d}} \alpha_{i} \tilde{x}_{i}(r)=0 \quad\left(\forall r \in R_{\epsilon_{1} \epsilon_{2} \epsilon_{3} \epsilon_{4} \epsilon_{5}}(b, d)\right)$.

Proof. $\quad$ - (1) $\Rightarrow(2)$. This is obvious.

- (1) $\Leftarrow(2)$. Let $r \in R_{\epsilon_{1} \epsilon_{2} \epsilon_{3} \epsilon_{4} \epsilon_{5}}$. By Lemma 1 .

$$
\sum_{n_{b-1}+1 \leq i \leq n_{d}} \alpha_{i} \tilde{x}_{i}(r)=\sum_{n_{b-1}+1 \leq i \leq n_{d}} \alpha_{i} \tilde{x}_{i}\left(O_{b, d}(r)\right) .
$$

By condition (2),

$$
\sum_{n_{b-1}+1 \leq i \leq n_{d}} \alpha_{i} \tilde{x}_{i}\left(O_{b, d}(r)\right)=0 .
$$


Then,

$$
\sum_{n_{b-1}+1 \leq i \leq n_{d}} \alpha_{i} \tilde{x}_{i}(r)=0
$$

We note that it is a simple task to give an explicit presentation of $R_{\epsilon_{1} \epsilon_{2} \epsilon_{3} \epsilon_{4} \epsilon_{5}}(b, d)$. In particular, if $d \leq 4$, one can do it by hands. The next example shows the concrete such process for a certain case.

Example 2 $\left(R_{00010}(2,3)\right)$. $R_{00010}(2,3)=\{\otimes+3 \otimes-3 \mathbb{Q}-\mathbb{Q}, 3 \otimes-2 \mathbb{D}-$ $\bigotimes, \bigotimes+\bigoplus-2 \otimes\}$. This fact is confirmed as follows.

Recall that a relator of Type (SIII) is of the form $r=([S i j T k i U j k]+[S i j T i U j]+$ $[S i T k i U k]+[S j T k U j k])-([S j i T i k U k j]+[$ SjiTiUj] $]+[$ SiTikUk] $]+[$ SjTkUkj] $)$. We immediately see, from this expression, that each term of $r$ consists of $n$ or $n+1$ letters for some $n$. Note that $O_{2,3}(r)$ is obtained from $r$ by removing the terms of length $<2$, or $>3$. Hence it is enough to suppose that each term of $r$ consists of two or three letters (Case 1) or each term of $r$ consists of three or four letters (Case 2).

- Case 1. Each term of $r$ consists of two or three letters.

In this case, we have $S T U=\emptyset$ (i.e., $S=\emptyset, T=\emptyset$, and $U=\emptyset$ ), and, hence,

$$
\begin{aligned}
O_{2,3}(r) & =r=[i j k i j k]+[i j i j]+[i k i k]+[j k j k]-[j i i k k j]-[j i i j]-[i i k k]-[j k k j] \\
& =[i j k i j k]+3[i j i j]-3[i i j j]-[i i j j k k] .
\end{aligned}
$$

- Case 2. Each term of $r$ consists of three or four letters.

In this case, $S T U=\sigma \sigma$ for some letter $\sigma(\neq i, j, k)$.

Case 2.1. Both $\sigma$ 's are contained in one of the sub-words $S, T$, and $U$.

Suppose that both $\sigma$ 's are contained in $S$. Then,

$$
\begin{aligned}
& r= {[\sigma \sigma i j k i j k]+[\sigma \sigma i j i j]+[\sigma \sigma i k i k]+[\sigma \sigma j k j k]-[\sigma \sigma j i i k k j]-[\sigma \sigma j i i j] } \\
& \quad-[\sigma \sigma i i k k]-[\sigma \sigma j k k j] \\
&=[\sigma \sigma i j k i j k]-[\sigma \sigma j i i k k j]+3[i j i j k k]-2[i j k k j i]-[i i j j k k] .
\end{aligned}
$$

Hence,

$$
O_{2,3}(r)=3[i j i j k k]-2[i j k k j i]-[i i j j k k] .
$$

By using similar arguments, we can show that we obtain the same element as above in other cases. Details of the calculations are left to the reader.

Case 2.2. The two $\sigma$ 's are contained in mutually different sub-words $S, T, U$.

Suppose that one $\sigma$ is contained in $S$ and the other $\sigma$ is contained in $T$. Then

$$
\begin{aligned}
r=[ & \sigma i j \sigma k i j k]+[\sigma i j \sigma i j]+[\sigma i \sigma k i k]+[\sigma j \sigma k j k]-[\sigma j i \sigma i k k j]-[\sigma j i \sigma i j] \\
& -[\sigma i \sigma i k k]-[\sigma j \sigma k k j] .
\end{aligned}
$$

Here, we note that

and

$$
[\sigma i \sigma i k k]=[\sigma j \sigma k k j]=[i j i j k k]
$$

These show that

$$
[\sigma i \sigma k i k]=[\sigma j \sigma k j k]=[\sigma j i \sigma i j]=[i j k i k j] .
$$

$$
r=[\sigma i j \sigma k i j k]-[\sigma j i \sigma i k k j]+[i j k i j k]+[i j k i k j]-2[i j i j k k] .
$$

Hence,

$$
O_{2,3}(r)=[i j k i j k]+[i j k i k j]-2[i j i j k k] .
$$


By using similar arguments, we can show that we obtain the same element as above in other cases. Details of the calculations are left to the reader.

As a conclusion, $R_{00010}(2,3)=\{[i j k i j k]+3[i j i j]-3[i i j j]-[j i i k k j], 3[i j i j k k]-$ $2[i j k k j i]-[i i j j k k],[i j k i j k]+[i j k i k j]-2[i j i j k k]\}=\{\otimes+3 \otimes-3 \mathbb{O}-\mathbb{O}, 3 \otimes-$ $2 \mathbb{D}-\mathbb{O}, \bigotimes+\bigoplus-2 \otimes\}$.

Example $3\left(R_{00010}(2,4)\right)$. By using the arguments in Example 2, we can show: $R_{00010}(2,4)=\{\otimes+3 \otimes-3 \mathbb{Q}-\mathbb{O}, \theta+3 \otimes-\ominus-2 \mathbb{D}-\mathbb{Q}, \bigotimes+\bigotimes+$ $\oplus-\mathbb{D}-2 \otimes, 2 \otimes-\otimes-\otimes, \bigotimes+\bigoplus-\otimes-2 \bigotimes, \otimes+2 \mathbb{Q}-\otimes-2 \otimes$,

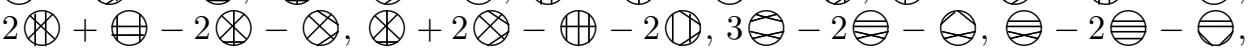
$\theta-2 \theta-0, \theta+\otimes-\theta-2 \theta$. Details of the proof are left to the reader.

Example $4\left(R_{00001}(2,3)\right)$. By using the arguments in Example 2 we can show:

$R_{00001}(2,3)=\{\oplus+\otimes-\mathbb{O}-\otimes, \otimes-\Theta, 3 \bigoplus-\otimes-2 \otimes, \otimes-2 \mathbb{D}+\mathbb{Q}, \otimes-\mathbb{O}\}$.

Details of the proof are left to the reader.

\subsection{Main result and corollaries.}

Theorem 1. Let $b$ and $d(2 \leq b \leq d)$ be integers and $G_{\leq d},\left\{x_{i}\right\}_{i \in \mathbb{N}}, \mathbb{Z}\left[G_{\leq l}\right], n_{d}=$ $\left|G_{\leq d}\right|$, and $G_{b, d}=\left\{x_{i}\right\}_{n_{b-1}+1 \leq i \leq n_{d}}$ be as in Subsection 2.1. Let $\sum_{n_{b-1}+1 \leq i \leq n_{d}} \alpha_{i} x_{i}$ and $\sum_{n_{b-1}+1 \leq i \leq n_{d}} \alpha_{i} \tilde{x}_{i}$ be functions as in Definition 6 . For $\left(\epsilon_{1}, \epsilon_{2}, \epsilon_{3}, \epsilon_{4}, \epsilon_{5}\right) \in$ $\{0,1\}^{5}$, let $R_{\epsilon_{1} \epsilon_{2} \epsilon_{3} \epsilon_{4} \epsilon_{5}}(b, d)$ be as in Subsection 2.2. Suppose the following conditions are satisfied:

- If $\epsilon_{1}=1, \sum_{n_{b-1}+1 \leq i \leq n_{d}} \alpha_{i} \tilde{x}_{i}(r)=0$ for each $r \in R_{10000}(b, d)$.

- If $\epsilon_{2}=1, \sum_{n_{b-1}+1 \leq i \leq n_{d}} \alpha_{i} \tilde{x}_{i}(r)=0$ for each $r \in R_{01000}(b, d)$.

- If $\epsilon_{3}=1, \sum_{n_{b-1}+1 \leq i \leq n_{d}} \alpha_{i} \tilde{x}_{i}(r)=0$ for each $r \in R_{00100}(b, d)$.

- If $\epsilon_{4}=1, \sum_{n_{b-1}+1 \leq i \leq n_{d}} \alpha_{i} \tilde{x}_{i}(r)=0$ for each $r \in R_{00010}(b, d)$.

- If $\epsilon_{5}=1, \sum_{n_{b-1}+1 \leq i \leq n_{d}} \alpha_{i} \tilde{x}_{i}(r)=0$ for each $r \in R_{00001}(b, d)$.

Then, the function $\sum_{n_{b-1}+1 \leq i \leq n_{d}} \alpha_{i} x_{i}$ on the set of ambient isotopy classes of spherical/plane curves is invariant under the Reidemeister moves corresponding to $\epsilon_{j}=1$.

Definition 8 (irreducible chord diagram). Let $x$ be a chord diagram. A chord $\alpha$ in $x$ is said to be an isolated chord if $\alpha$ does not intersect any other chord. If $x$ has an isolated chord, $x$ is called reducible and otherwise, $x$ is called irreducible. The set of the irreducible chord diagrams is denoted by Irr. Let $I_{b, d}^{(\operatorname{Irr})}=\left\{i \mid n_{b-1}+1 \leq\right.$ $\left.i \leq n_{d}, x_{i} \in \operatorname{Irr}\right\}$.

If we consider the function of the form $\sum_{i \in I_{b, d}^{(\mathrm{Irr})}} \alpha_{i} x_{i}$ for $\sum_{n_{b-1}+1 \leq i \leq n_{d}} \alpha_{i} x_{i}$ in Theorem 1, we obtain:

Corollary 1. Let $b$ and $d(2 \leq b \leq d)$ be integers and $G_{\leq d},\left\{x_{i}\right\}_{i \in \mathbb{N}}, \mathbb{Z}\left[G_{\leq l}\right], n_{d}$ $=\left|G_{\leq d}\right|$, and $G_{b, d}=\left\{x_{i}\right\}_{n_{b-1}+1 \leq i \leq n_{d}}$ be as in Subsection 2.1. Let $\sum_{i \in I_{b, d}^{(\mathrm{Irr})}} \alpha_{i} x_{i}$ 
and $\sum_{i \in I_{b, d}^{(\mathrm{Irr})}} \alpha_{i} \tilde{x}_{i}$ be functions as in Definition $\sqrt{6}$. For $\left(\epsilon_{1}, \epsilon_{2}, \epsilon_{3}, \epsilon_{4}, \epsilon_{5}\right) \in\{0,1\}^{5}$, let $R_{\epsilon_{1} \epsilon_{2} \epsilon_{3} \epsilon_{4} \epsilon_{5}}(b, d)$ be as in Subsection 2.2. Suppose the following conditions are satisfied:

- If $\epsilon_{2}=1, \sum_{i \in I_{b, d}^{(\mathrm{Irr})}} \alpha_{i} \tilde{x}_{i}(r)=0$ for each $r \in R_{01000}(b, d)$.

- If $\epsilon_{3}=1, \sum_{i \in I_{b, d}^{(\mathrm{Irr})}} \alpha_{i} \tilde{x}_{i}(r)=0$ for each $r \in R_{00100}(b, d)$.

- If $\epsilon_{4}=1, \sum_{i \in I_{b, d}^{(\mathrm{Irr})}} \alpha_{i} \tilde{x}_{i}(r)=0$ for each $r \in R_{00010}(b, d)$.

- If $\epsilon_{5}=1, \sum_{i \in I_{b, d}^{(\mathrm{Irr})}} \alpha_{i} \tilde{x}_{i}(r)=0$ for each $r \in R_{00001}(b, d)$.

Then, the function $\sum_{i \in I_{b, d}^{(\mathrm{Irr})}} \alpha_{i} x_{i}$ on the set of ambient isotopy classes of spheri$\mathrm{cal} /$ plane curves is invariant under RI and the Reidemeister moves corresponding to $\epsilon_{j}=1$.

Proof of Corollary 1 from Theorem 1. By Theorem 1, it is enough to show

$$
\sum_{i \in I_{b, d}^{(\mathrm{Irr})}} \alpha_{i} \tilde{x}_{i}(r)=0 \quad\left(\forall r \in R_{10000}(b, d)\right)
$$

for a proof of Corollary 1. We first note that if $x_{i} \in \operatorname{Irr}$, then $x_{i}$ has no isolated chords. On the other hand, let $r \in R_{10000}(b, d)$, that is, there exist a Gauss word $S$ and a letter $j$ such that $r=[S j j]$. Then, the chord corresponding to $j$ is isolated. These show that $\tilde{x}_{i}(r)=0$. This shows that $(2.4)$ holds.

Note that $\sum_{i \in I_{b, d}^{(\mathrm{Irr})}} \alpha_{i} x_{i}$ is a special case of $\sum_{n_{b-1}+1 \leq j \leq n_{d}} \alpha_{j} x_{j}$. Thus, we may suppose that integers $\alpha_{j}\left(n_{b-1}+1 \leq j \leq n_{d}\right)$ satisfy that $\sum_{i \in I_{b, d}^{(\operatorname{Irr})}} \alpha_{i} x_{i}=$ $\sum_{n_{b-1}+1 \leq j \leq n_{d}} \alpha_{j} x_{j}$. Therefore, if $\sum_{i \in I_{b, d}^{(\operatorname{Irr})}} \alpha_{i} \tilde{x}_{i}(r)=0$, then $\sum_{n_{b-1}+1 \leq j \leq n_{d}} \alpha_{j} \tilde{x}_{j}(r)$ $=0$. By Theorem 1. $\sum_{n_{b-1}+1 \leq j \leq n_{d}} \alpha_{j} x_{j}$ is invariant. Since each non-zero term is common, $\sum_{i \in I_{b, d}^{(\mathrm{Irr})}} \alpha_{i} x_{i}$ is also invariant.

Example 5. It is easy to see that $\left\{x_{i}\right\}_{i \in I_{2,3}^{(\operatorname{Irr})}}$ consists of three elements, $y_{1}=$ $\otimes, y_{2}=\bigotimes$, and $y_{3}=\bigoplus$. This fact together with Corollary 1 implies that if there exist integers $\alpha_{1}, \alpha_{2}$, and $\alpha_{3}$ such that $\left(\alpha_{1} \tilde{y}_{1}+\alpha_{2} \tilde{y}_{2}+\alpha_{3} \tilde{y}_{3}\right)(r)=0$ $\left(\forall r \in R_{00010}(2,3)\right)$, then $\alpha_{1} y_{1}+\alpha_{2} y_{2}+\alpha_{3} y_{3}$ is invariant under RI and strong RIII. Recall that $R_{00010}(2,3)$ consists of the following three elements $r_{1}, r_{2}$, and $r_{3}$ (Example 2):

$$
\begin{aligned}
& r_{1}=\bigotimes+3 \otimes-3 \mathbb{O}-\mathbb{O}, \\
& r_{2}=3 \otimes-2 \mathbb{D}-\mathbb{Q}, \text { and } \\
& r_{3}=\bigoplus+\bigoplus-2 \otimes .
\end{aligned}
$$

We can find such $\alpha_{1}, \alpha_{2}$, and $\alpha_{3}$ by solving the linear equation $\mathbf{x} M=\mathbf{0}$, where $M=\left(\tilde{y}_{i}\left(r_{j}\right)\right)$ (Figure 9). It is elementary to show that the set of solutions is $\{\mathbf{x}=$ $\gamma(1,-3,3) \mid \gamma \in \mathbb{Z}\}$. Particularly, $y_{1}-3 y_{2}+3 y_{3}$ is invariant under RI and strong RIII. We note that $\frac{1}{4}\left(y_{1}-3 y_{2}+3 y_{3}\right)$ is the function $\lambda$ introduced in [5] from a different view point. 
Example 6. In contrast to Example 5, we will note that there does not exist a nonzero $\left(\alpha_{1}, \alpha_{2}, \alpha_{3}\right)$ such that $\left(\alpha_{1} \tilde{y}_{1}+\alpha_{2} \tilde{y}_{2}+\alpha_{3} \tilde{y}_{3}\right)(r)=0$ for each $r \in$ $R_{00001}(2,3)$, i.e., there does not exist $\alpha_{1} \tilde{y}_{1}+\alpha_{2} \tilde{y}_{2}+\alpha_{3} \tilde{y}_{3}$ satisfying the condition of Corollary 1 with $\epsilon_{5}=1$. In fact, we take $y_{i}(i=1,2,3)$ as in Example 5 and name the elements of $R_{00001}(2,3)$ (see Example 4 ) as:

$$
\begin{aligned}
& r_{1}^{\prime}=\bigoplus+\otimes-\mathbb{D}-\otimes, \\
& r_{2}^{\prime}=\otimes-\otimes, \\
& r_{3}^{\prime}=3 \bigoplus-\otimes-2 \otimes, \\
& r_{4}^{\prime}=\otimes-2 \mathbb{D}+\mathbb{Q}, \text { and } \\
& r_{5}^{\prime}=\otimes-\mathbb{O} .
\end{aligned}
$$

Let $M=\left(\tilde{y}_{i}\left(r_{j}^{\prime}\right)\right)_{1 \leq i \leq 3,1 \leq j \leq 5}$ (Figure 10 . Then it is easy to see that the linear equation $\mathbf{x} M=\mathbf{0}$ admits the trivial solution $\mathbf{x}=(0,0,0)$ only.

Definition 9 (connected chord diagram). Let $v$ be a Gauss word of length $2 m$ and $w$ a Gauss word of length $2 n$ where $v(2 \hat{m}) \cap w(\hat{2 n})=\emptyset$. Then we define the Gauss word of length $2(m+n)$, denoted by $v w$, by $v w(i)=v(i)(1 \leq i \leq 2 m)$ and $v w(2 m+i)=w(i)(1 \leq i \leq 2 n)$. The chord diagram $[v w]$ is called a product of chord diagrams $[v]$ and $[w]$. If a chord diagram is not a product of two non-empty chord diagrams, then the chord diagram is called a connected chord diagram. The set of the connected chord diagrams is denoted by Conn. Let $I_{b, d}^{(\mathrm{Conn})}=\left\{i \mid n_{b-1}+1 \leq\right.$ $i \leq n_{d}, x_{i} \in$ Conn $\}$.

It is easy to see that if a connected chord diagram has at least two chords, it is irreducible. Note that the chord diagram consisting of exactly one chord is connected but not irreducible.

Recall that $\mathcal{C}$ is the set of ambient isotopy classes of spherical/plane curves. In Definition 10, for plane curves, we freely use the identification of $S^{2}$ with $\mathbb{R}^{2} \cup\{\infty\}$.

Definition 10 (connected sum). Let $P_{1}, P_{2} \in \mathcal{C}$. We suppose that the ambient 2 -spheres are oriented. Let $p_{i}$ be a point on $P_{i}$ such that $p_{i}$ is not a double point $(i=1,2)$. Let $d_{i}$ be a sufficiently small disk with center $p_{i}(i=1,2)$ such that $d_{i} \cap P_{i}$ consists of an arc properly embedded in $d_{i}$. Let $\hat{d}_{i}=\operatorname{cl}\left(S^{2} \backslash d_{i}\right)$ and $\hat{P}_{i}=P_{i} \cap \hat{d}_{i}$; let $h: \partial \hat{d}_{1} \rightarrow \partial \hat{d}_{2}$ be an orientation reversing homeomorphism such that $h\left(\partial \hat{P}_{1}\right)$ $=\partial \hat{P}_{2}$. Then, $\hat{P}_{1} \cup_{h} \hat{P}_{2}$ gives a spherical curve in the oriented 2-sphere $\hat{d}_{1} \cup_{h} \hat{d}_{2}$.

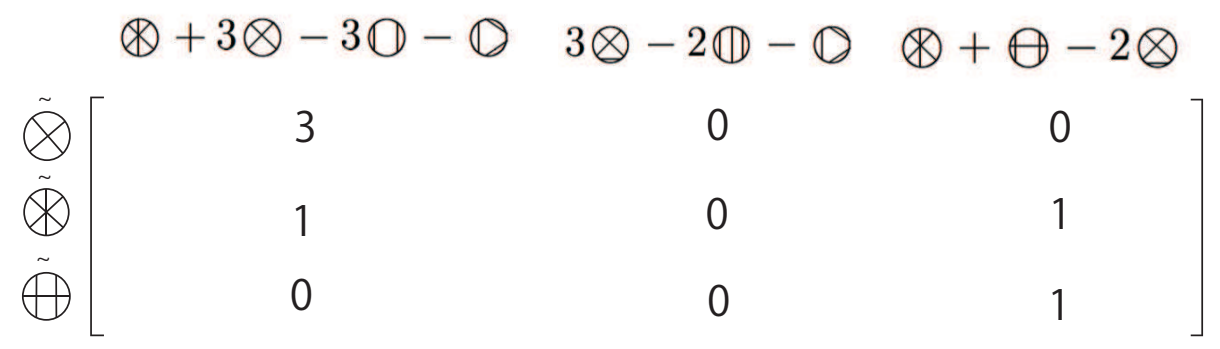

Figure 9. Matrix $\left(\tilde{y}_{i}\left(r_{j}\right)\right)_{1 \leq i, j \leq 3}$. 
NOBORU ITO

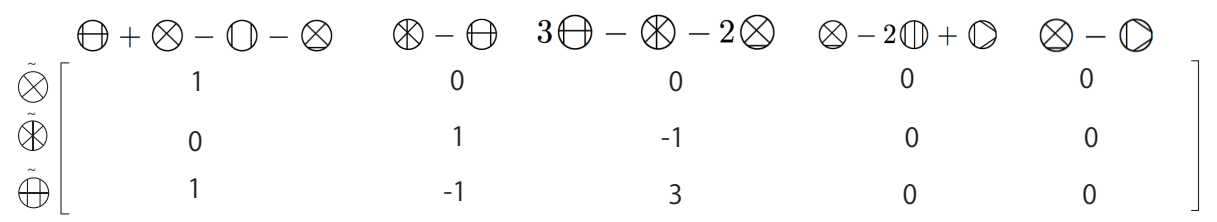

FiguRE 10. Matrix $\left(\tilde{y}_{i}\left(r_{j}^{\prime}\right)\right)_{1 \leq i \leq 3,1 \leq j \leq 5}$.

The spherical curve $\hat{P}_{1} \cup_{h} \hat{P}_{2}$ in the oriented 2 -sphere, denoted by $P_{1} \sharp\left(p_{1}, p_{2}\right), h P_{2}$, is called a connected sum of the spherical/plane curves $P_{1}$ and $P_{2}$ at the pair of points $p_{1}$ and $p_{2}$ (see Figure 11).

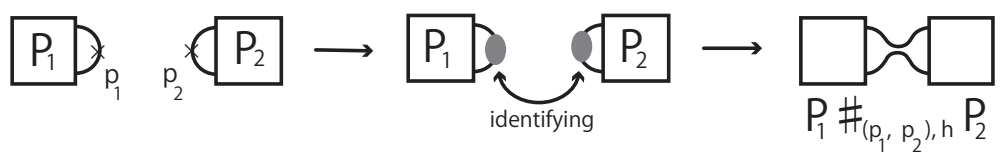

Figure 11. Connected sum $P_{1} \sharp\left(p_{1}, p_{2}\right), h P_{2}$ of two spherical/plane curves $P_{1}$ and $P_{2}$.

Definition 11 (additivity). Let $I$ be a $\mathbb{Z}$-valued function on $\mathcal{C}$. We say that $I$ is additive if $I\left(P_{1} \sharp\left(p_{1}, p_{2}\right), h P_{2}\right)=I\left(P_{1}\right)+I\left(P_{2}\right)$ for any spherical/plane curves $P_{1}$ and $P_{2}$ and for any $P_{1} \sharp\left(p_{1}, p_{2}\right), h P_{2}$ in Definition 10

Definition 12 (prime). A spherical/plane curve $P$ is trivial if $P$ is a simple closed curve. A spherical/plane curve $P$ is prime if $P$ cannot be expressed as a connected sum of two nontrivial spherical/plane curves.

If we consider the function of the form $\sum_{i \in I_{b, d}^{(\mathrm{Conn})}} \alpha_{i} x_{i}$ for $\sum_{n_{b-1}+1 \leq i \leq n_{d}} \alpha_{i} x_{i}$ in Theorem 1, we obtain:

Corollary 2. Let $b$ and $d(2 \leq b \leq d)$ be integers and $G_{\leq d},\left\{x_{i}\right\}_{i \in \mathbb{N}}, \mathbb{Z}\left[G_{\leq l}\right], n_{d}$ $=\left|G_{\leq d}\right|$, and $G_{b, d}=\left\{x_{i}\right\}_{n_{b-1}+1 \leq i \leq n_{d}}$ be as in Subsection 2.1. Let $\sum_{i \in I_{b, d}^{(\mathrm{Conn})}} \alpha_{i} x_{i}$ and $\sum_{i \in I_{b, d}^{(\mathrm{Conn})}} \alpha_{i} \tilde{x}_{i}$ be functions as in Definition $\sqrt{6}$. For $\left(\epsilon_{1}, \epsilon_{2}, \epsilon_{3}, \epsilon_{4}, \epsilon_{5}\right) \in\{0,1\}^{5}$, let $R_{\epsilon_{1} \epsilon_{2} \epsilon_{3} \epsilon_{4} \epsilon_{5}}(b, d)$ be as in Subsection 2.2. Suppose the following conditions are satisfied:

- If $\epsilon_{2}=1, \sum_{i \in I_{b, d}^{(\text {Conn })}} \alpha_{i} \tilde{x}_{i}(r)=0$ for each $r \in R_{01000}(b, d)$.

- If $\epsilon_{3}=1, \sum_{i \in I_{b, d}^{\text {(Conn) }}} \alpha_{i} \tilde{x}_{i}(r)=0$ for each $r \in R_{00100}(b, d)$.

- If $\epsilon_{4}=1, \sum_{i \in I_{b, d}^{(\mathrm{Conn})}} \alpha_{i} \tilde{x}_{i}(r)=0$ for each $r \in R_{00010}(b, d)$.

- If $\epsilon_{5}=1, \sum_{i \in I_{b, d}^{(\mathrm{Conn})}} \alpha_{i} \tilde{x}_{i}(r)=0$ for each $r \in R_{00001}(b, d)$. 
Then, the function $\sum_{i \in I_{b, d}^{(\mathrm{Conn})}} \alpha_{i} x_{i}$ on the set of ambient isotopy classes of spherical/plane curves is additive and invariant under RI and the Reidemeister moves corresponding to $\epsilon_{j}=1$.

Proof of Corollary 2 from Theorem 1. Since $b \geq 2$ and $i \geq n_{b-1}+1$, each $x_{i}$ consists of more than one chords, i.e., $x_{i} \in \operatorname{Irr}$ (see the note preceding Definition 10 ).

By Theorem 1, it is enough to show

$$
\sum_{i \in I_{b, d}^{(\mathrm{Conn})}} \alpha_{i} \tilde{x}_{i}(r)=0 \quad\left(\forall r \in R_{10000}(b, d)\right)
$$

for a proof of Corollary 2. We first note that if $x_{i} \in \operatorname{Irr}$, then $x_{i}$ has no isolated chords. On the other hand, let $r \in R_{10000}(b, d)$, that is, there exist a Gauss word $S$ and a letter $j$ such that $r=[S j j]$. Then, the chord corresponding to $j$ is isolated. These show that $\tilde{x}_{i}(r)=0$. This shows that 2.5 holds.

Note that $\sum_{i \in I_{b, d}^{(\text {Conn })}} \alpha_{i} x_{i}$ is a special case of $\sum_{n_{b-1}+1 \leq j \leq n_{d}} \alpha_{j} x_{j}$. Thus, we may suppose that integers $\alpha_{j}\left(n_{b-1}+1 \leq j \leq n_{d}\right)$ satisfy that $\sum_{i \in I_{b, d}^{(\text {Conn })}} \alpha_{i} x_{i}=$ $\sum_{n_{b-1}+1 \leq j \leq n_{d}} \alpha_{j} x_{j}$. Therefore, if $\sum_{i \in I_{b, d}^{(\mathrm{Conn})}} \alpha_{i} \tilde{x}_{i}(r)=0$, then $\sum_{n_{b-1}+1 \leq j \leq n_{d}} \alpha_{j} \tilde{x}_{j}(r)$ $=0$. By Theorem 1 $\sum_{n_{b-1}+1 \leq j \leq n_{d}} \alpha_{j} x_{j}$ is invariant. Since each non-zero term is common, $\sum_{i \in I_{b, d}^{(\mathrm{Conn})}} \alpha_{i} x_{i}$ is also invariant.

Further, by using geometric observations as in Example 1 it is clear that if $x_{i} \in$ Conn, then

$$
x_{i}\left(P_{1} \sharp\left(p_{1}, p_{2}\right), h P_{2}\right)=x_{i}\left(P_{1}\right)+x_{i}\left(P_{2}\right)
$$

for any $P_{1} \sharp\left(p_{1}, p_{2}\right), h P_{2}$. This fact implies $\sum_{i \in I_{b, d}^{(\mathrm{Conn})}} \alpha_{i} x_{i}$ is additive.

\section{Proof of Theorem 1}

Since every Reidemeister move on plane curves may be regarded as that of spherical curves, we will prove the statement for Reidemeister moves on spherical curves in the following.

- (Proof of the case $\epsilon_{1}=1$.) Let $P$ and $P^{\prime}$ be two spherical curves where $P$ and $P^{\prime}$ are related by a single RI, hence, there exist a letter $i$ and two Gauss words $G=S i i$ and $G^{\prime}=S$ corresponding to $P$ and $P^{\prime}$, respectively, i.e., $C D_{P}=$ $[S i i]$ and $C D_{P^{\prime}}=[S]$. As we observed in a paragraph preceding of Definition 7 in Subsection 2.2, we have

$$
\sum_{n_{b-1}+1 \leq i \leq n_{d}} \alpha_{i} x_{i}(P)-\sum_{n_{b-1}+1 \leq i \leq n_{d}} \alpha_{i} x_{i}\left(P^{\prime}\right)=\sum_{n_{b-1}+1 \leq i \leq n_{d}} \sum_{z_{0} \in \operatorname{Sub}^{(0)}(G)} \alpha_{i} \tilde{x}_{i}\left(\left[z_{0} i i\right]\right) .
$$

By the assumption of this case, for each $z_{0} \in \operatorname{Sub}^{(0)}(G)$,

$$
\sum_{n_{b-1}+1 \leq i \leq n_{d}} \alpha_{i} \tilde{x}_{i}\left(\left[z_{0} i i\right]\right)=0
$$

and this shows that

$$
\sum_{n_{b-1}+1 \leq i \leq n_{d}} \alpha_{i} x_{i}(P)=\sum_{n_{b-1}+1 \leq i \leq n_{d}} \alpha_{i} x_{i}\left(P^{\prime}\right)
$$

Hence, $\sum_{n_{b-1}+1 \leq i \leq n_{d}} \alpha_{i} x_{i}$ is invariant under RI. 
- (Proof of the case $\epsilon_{2}=1$.) Let $P$ and $P^{\prime}$ be two spherical curves where $P$ and $P^{\prime}$ are related by a single strong RII, hence, there exist two Gauss words $G=\operatorname{SijTji}$ and $G^{\prime}=S T$ corresponding to $P$ and $P^{\prime}$, respectively, i.e., $C D_{P}=[S i j T j i]$ and $C D_{P^{\prime}}=[S T]$.

By (2.1) in Subsection 2.1 and 2.2) in Subsection 2.2 we obtain (note that $\left.\operatorname{Sub}^{(3)}(G)=\emptyset\right)$ :

$$
\begin{aligned}
& \sum_{n_{b-1}+1 \leq i \leq n_{d}} \alpha_{i} x_{i}(P)=\sum_{n_{b-1}+1 \leq i \leq n_{d}} \alpha_{i}\left(\sum_{z \in \operatorname{Sub}(G)} \tilde{x}_{i}(z)\right) \\
= & \sum_{n_{b-1}+1 \leq i \leq n_{d}} \alpha_{i}\left(\sum_{z_{0} \in \operatorname{Sub}^{(0)}(G)} \tilde{x}_{i}\left(z_{0}\right)\right)+\sum_{n_{b-1}+1 \leq i \leq n_{d}} \sum_{z_{12} \in \operatorname{Sub}^{(1)}(G) \cup \operatorname{Sub}^{(2)}(G)} \alpha_{i} \tilde{x}_{i}\left(z_{12}\right) \\
= & \sum_{n_{b-1}+1 \leq i \leq n_{d}} \alpha_{i}\left(\sum_{z^{\prime} \in \operatorname{Sub}\left(G^{\prime}\right)} \tilde{x}_{i}\left(z^{\prime}\right)\right)+\sum_{n_{b-1}+1 \leq i \leq n_{d} z_{12} \in \operatorname{Sub}^{(1)}(G) \cup \operatorname{Sub}^{(2)}(G)} \alpha_{i} \tilde{x}_{i}\left(z_{12}\right)
\end{aligned}
$$

$\left(\because \operatorname{Sub}^{(0)}(G)\right.$ is identified with $\left.\operatorname{Sub}\left(G^{\prime}\right)\right)$.

Let $z_{0} \in \operatorname{Sub}^{(0)}(G)$. We note that since $G$ is a Gauss word $z_{0}$ uniquely admits a decomposition into two sub-words, which are sub-words on $S$ and $T$. Let $\sigma\left(z_{0}\right)$ be the sub-word of $S$ and $\tau\left(z_{0}\right)$ the sub-word of $T$ satisfying $z_{0}=\sigma\left(z_{0}\right) \tau\left(z_{0}\right)$. Under these notations, we define maps

$$
\begin{aligned}
& z_{2}: \operatorname{Sub}^{(0)}(G) \rightarrow \operatorname{Sub}^{(2)}(G) ; z_{2}\left(z_{0}\right)=\sigma\left(z_{0}\right) i j \tau\left(z_{0}\right) j i, \\
& z_{1}: \operatorname{Sub}^{(0)}(G) \rightarrow \operatorname{Sub}^{(1)}(G) ; z_{1}\left(z_{0}\right)=\sigma\left(z_{0}\right) i \tau\left(z_{0}\right) i, \text { and } \\
& z_{1}^{\prime}: \operatorname{Sub}^{(0)}(G) \rightarrow \operatorname{Sub}^{(1)}(G) ; z_{1}^{\prime}\left(z_{0}\right)=\sigma\left(z_{0}\right) j \tau\left(z_{0}\right) j .
\end{aligned}
$$

Then, it is easy to see that $\operatorname{Sub}^{(1)}(G) \cup \operatorname{Sub}^{(2)}(G)$ admits a decomposition

$$
\begin{aligned}
& \operatorname{Sub}^{(1)}(G) \cup \operatorname{Sub}^{(2)}(G) \\
& =\left\{z_{1}\left(z_{0}\right) \mid z_{0} \in \operatorname{Sub}^{(0)}(G)\right\} \amalg\left\{z_{1}^{\prime}\left(z_{0}\right) \mid z_{0} \in \operatorname{Sub}^{(0)}(G)\right\} \amalg\left\{z_{2}\left(z_{0}\right) \mid z_{0} \in \operatorname{Sub}^{(0)}(G)\right\} .
\end{aligned}
$$

These notations together with the above give:

$$
\begin{aligned}
\sum_{n_{b-1}+1 \leq i \leq n_{d}} \alpha_{i} x_{i}(P)= & \sum_{n_{b-1}+1 \leq i \leq n_{d}} \alpha_{i} x_{i}\left(P^{\prime}\right) \\
& +\sum_{n_{b-1}+1 \leq i \leq n_{d}} \sum_{z_{0} \in \operatorname{Sub}^{(0)}(G)} \alpha_{i} \tilde{x}_{i}\left(z_{1}\left(z_{0}\right)+z_{1}^{\prime}\left(z_{0}\right)+z_{2}\left(z_{0}\right)\right) \\
= & \sum_{n_{b-1}+1 \leq i \leq n_{d}} \alpha_{i} x_{i}\left(P^{\prime}\right) \\
& +\sum_{n_{b-1}+1 \leq i \leq n_{d}} \sum_{z_{0} \in \operatorname{Sub}^{(0)}(G)} \alpha_{i} \tilde{x}_{i}\left(\left[z_{1}\left(z_{0}\right)\right]+\left[z_{1}^{\prime}\left(z_{0}\right)\right]+\left[z_{2}\left(z_{0}\right)\right]\right) .
\end{aligned}
$$

By Proposition 1 and by the condition for the case $\epsilon_{2}=1$, for any $z_{0} \in \operatorname{Sub}^{(0)}(G)$,

$$
\sum_{n_{b-1}+1 \leq i \leq n_{d}} \alpha_{i} \tilde{x}_{i}\left(\left[z_{1}\left(z_{0}\right)\right]+\left[z_{1}^{\prime}\left(z_{0}\right)\right]+\left[z_{2}\left(z_{0}\right)\right]\right)=0 .
$$


Thus,

$$
\sum_{n_{b-1}+1 \leq i \leq n_{d}} \alpha_{i} x_{i}(P)=\sum_{n_{b-1}+1 \leq i \leq n_{d}} \alpha_{i} x_{i}\left(P^{\prime}\right)
$$

Hence, $\sum_{n_{b-1}+1 \leq i \leq n_{d}} \alpha_{i} x_{i}$ is invariant under strong RII.

- (Proof of the case $\epsilon_{3}=1$.) Since the arguments are essentially the same as those for the case $\epsilon_{2}=1$, we omit this proof.

- (Proof of the case $\epsilon_{4}=1$.) Let $P$ and $P^{\prime}$ be two spherical curves where $P$ and $P^{\prime}$ are related by a single strong RIII, hence, there exist two Gauss words $G=S i j T k i U j k$ and $G^{\prime}=S j i T i k U k j$ corresponding to $P$ and $P^{\prime}$, respectively, i.e., $C D_{P}=[S i j T k i U j k]$ and $C D_{P^{\prime}}=[$ SjiTikUkj].

By (2.1) in Subsection 2.1 and 2.2) in Subsection 2.2, we obtain:

$$
\begin{aligned}
& \sum_{n_{b-1}+1 \leq i \leq n_{d}} \alpha_{i} x_{i}(P)=\sum_{n_{b-1}+1 \leq i \leq n_{d}} \alpha_{i}\left(\sum_{z \in \operatorname{Sub}(G)} \tilde{x}_{i}(z)\right) \\
= & \sum_{n_{b-1}+1 \leq i \leq n_{d}} \alpha_{i}\left(\sum_{z_{01} \in \operatorname{Sub}^{(0)}(G) \cup \operatorname{Sub}^{(1)}(G)} \tilde{x}_{i}\left(z_{01}\right)+\sum_{z_{23} \in \operatorname{Sub}^{(2)}(G) \cup \operatorname{Sub}^{(3)}(G)} \tilde{x}_{i}\left(z_{23}\right)\right) .
\end{aligned}
$$

and

$$
\begin{aligned}
& \sum_{n_{b-1}+1 \leq i \leq n_{d}} \alpha_{i} x_{i}\left(P^{\prime}\right)=\sum_{n_{b-1}+1 \leq i \leq n_{d}} \alpha_{i}\left(\sum_{z^{\prime} \in \operatorname{Sub}\left(G^{\prime}\right)} \tilde{x}_{i}\left(z^{\prime}\right)\right) \\
= & \sum_{n_{b-1}+1 \leq i \leq n_{d}} \alpha_{i}\left(\sum_{z_{01}^{\prime} \in \operatorname{Sub}^{(0)}\left(G^{\prime}\right) \cup \operatorname{Sub}^{(1)}\left(G^{\prime}\right)} \tilde{x}_{i}\left(z_{01}^{\prime}\right)+\sum_{z_{23}^{\prime} \in \operatorname{Sub}^{(2)}\left(G^{\prime}\right) \cup \operatorname{Sub}^{(3)}\left(G^{\prime}\right)} \tilde{x}_{i}\left(z_{23}^{\prime}\right)\right) .
\end{aligned}
$$

Since $\operatorname{Sub}^{(0)}(G)\left(\operatorname{Sub}^{(1)}(G)\right.$ resp. $)$ is naturally identified with $\operatorname{Sub}^{(0)}\left(G^{\prime}\right)\left(\operatorname{Sub}^{(1)}\left(G^{\prime}\right)\right.$ resp.), the above equations show:

$$
\begin{aligned}
& \sum_{n_{b-1}+1 \leq i \leq n_{d}} \alpha_{i} x_{i}(P)-\sum_{n_{b-1}+1 \leq i \leq n_{d}} \sum_{z_{23} \in \operatorname{Sub}^{(2)}(G) \cup \operatorname{Sub}^{(3)}(G)} \alpha_{i} \alpha_{i} x_{i}\left(P^{\prime}\right) \\
& \quad-\sum_{n_{b-1}+1 \leq i \leq n_{d}\left(z_{23}\right)} \sum_{z_{23}^{\prime} \in \operatorname{Sub}^{(2)}\left(G^{\prime}\right) \cup \operatorname{Sub}^{(3)}\left(G^{\prime}\right)} \alpha_{i} \tilde{x}_{i}\left(z_{23}^{\prime}\right) .
\end{aligned}
$$

Let $z_{0} \in \operatorname{Sub}^{(0)}(G)$, which is identified with $\operatorname{Sub}^{(0)}\left(G^{\prime}\right)$. We note that since $G$ is a Gauss word $z_{0}$ uniquely admits a decomposition into three sub-words, which are sub-words on $S, T$, and $U$. Let $\sigma\left(z_{0}\right)$ be the sub-word of $S, \tau\left(z_{0}\right)$ the sub-word of $T$, and $\mu\left(z_{0}\right)$ the sub-word of $U$ satisfying $z_{0}=\sigma\left(z_{0}\right) \tau\left(z_{0}\right) \mu\left(z_{0}\right)$. We define maps

$$
\begin{aligned}
z_{3} & : \operatorname{Sub}^{(0)}(G) \rightarrow \operatorname{Sub}^{(3)}(G) ; z_{3}\left(z_{0}\right)=\sigma\left(z_{0}\right) i j \tau\left(z_{0}\right) k i \mu\left(z_{0}\right) j k, \\
z_{2 a} & : \operatorname{Sub}^{(0)}(G) \rightarrow \operatorname{Sub}^{(2)}(G) ; z_{2 a}\left(z_{0}\right)=\sigma\left(z_{0}\right) i j \tau\left(z_{0}\right) i \mu\left(z_{0}\right) j, \\
z_{2 b} & : \operatorname{Sub}^{(0)}(G) \rightarrow \operatorname{Sub}^{(2)}(G) ; z_{2 b}\left(z_{0}\right)=\sigma\left(z_{0}\right) i \tau\left(z_{0}\right) k i \mu\left(z_{0}\right) k, \text { and } \\
z_{2 c} & : \operatorname{Sub}^{(0)}(G) \rightarrow \operatorname{Sub}^{(2)}(G) ; z_{2 c}\left(z_{0}\right)=\sigma\left(z_{0}\right) j \tau\left(z_{0}\right) k \mu\left(z_{0}\right) j k .
\end{aligned}
$$


Similarly, let

$$
\begin{aligned}
& z_{3}^{\prime}: \operatorname{Sub}^{(0)}\left(G^{\prime}\right) \rightarrow \operatorname{Sub}^{(3)}\left(G^{\prime}\right) ; z_{3}^{\prime}\left(z_{0}\right)=\sigma\left(z_{0}\right) j i \tau\left(z_{0}\right) i k \mu\left(z_{0}\right) k j, \\
& z_{2 a}^{\prime}: \operatorname{Sub}^{(0)}\left(G^{\prime}\right) \rightarrow \operatorname{Sub}^{(2)}\left(G^{\prime}\right) ; z_{2 a}^{\prime}\left(z_{0}\right)=\sigma\left(z_{0}\right) j i \tau\left(z_{0}\right) i \mu\left(z_{0}\right) j, \\
& z_{2 b}^{\prime}: \operatorname{Sub}^{(0)}\left(G^{\prime}\right) \rightarrow \operatorname{Sub}^{(2)}\left(G^{\prime}\right) ; z_{2 b}^{\prime}\left(z_{0}\right)=\sigma\left(z_{0}\right) i \tau\left(z_{0}\right) i k \mu\left(z_{0}\right) k, \text { and } \\
& z_{2 c}^{\prime}: \operatorname{Sub}^{(0)}\left(G^{\prime}\right) \rightarrow \operatorname{Sub}^{(2)}\left(G^{\prime}\right) ; z_{2 c}^{\prime}\left(z_{0}\right)=\sigma\left(z_{0}\right) j \tau\left(z_{0}\right) k \mu\left(z_{0}\right) k j .
\end{aligned}
$$

Then, it is easy to see that $\operatorname{Sub}^{(2)}(G) \cup \operatorname{Sub}^{(3)}(G)$ admits decompositions $\operatorname{Sub}^{(2)}(G) \cup \operatorname{Sub}^{(3)}(G)$

$=\left\{z_{3}\left(z_{0}\right) \mid z_{0} \in \operatorname{Sub}^{(0)}(G)\right\} \amalg\left\{z_{2 a}\left(z_{0}\right) \mid z_{0} \in \operatorname{Sub}^{(0)}(G)\right\} \amalg\left\{z_{2 b}\left(z_{0}\right) \mid z_{0} \in \operatorname{Sub}^{(0)}(G)\right\}$

$\amalg\left\{z_{2 c}\left(z_{0}\right) \mid z_{0} \in \operatorname{Sub}^{(0)}(G)\right\}$

and

$\operatorname{Sub}^{(2)}\left(G^{\prime}\right) \cup \operatorname{Sub}^{(3)}\left(G^{\prime}\right)$

$=\left\{z_{3}^{\prime}\left(z_{0}\right) \mid z_{0} \in \operatorname{Sub}^{(0)}(G)\right\} \amalg\left\{z_{2 a}^{\prime}\left(z_{0}\right) \mid z_{0} \in \operatorname{Sub}^{(0)}(G)\right\} \amalg\left\{z_{2 b}^{\prime}\left(z_{0}\right) \mid z_{0} \in \operatorname{Sub}^{(0)}(G)\right\}$

$\amalg\left\{z_{2 c}^{\prime}\left(z_{0}\right) \mid z_{0} \in \operatorname{Sub}^{(0)}(G)\right\}$.

By using these notations, we obtain:

$$
\begin{aligned}
& \sum_{n_{b-1}+1 \leq i \leq n_{d}} \alpha_{i} x_{i}(P)-\sum_{n_{b-1}+1 \leq i \leq n_{d}} \alpha_{i} x_{i}\left(P^{\prime}\right) \\
= & \sum_{z_{0} \in \operatorname{Sub}^{(0)}(G)} \sum_{n_{b-1}+1 \leq i \leq n_{d}} \alpha_{i} \tilde{x}_{i}\left(\left(z_{3}\left(z_{0}\right)+z_{2 a}\left(z_{0}\right)+z_{2 b}\left(z_{0}\right)+z_{2 c}\left(z_{0}\right)\right)\right. \\
- & \left.\left(z_{3}^{\prime}\left(z_{0}\right)+z_{2 a}^{\prime}\left(z_{0}\right)+z_{2 b}^{\prime}\left(z_{0}\right)+z_{2 c}^{\prime}\left(z_{0}\right)\right)\right) \\
= & \sum_{z_{0} \in \operatorname{Sub}^{(0)}(G)} \sum_{n_{b-1}+1 \leq i \leq n_{d}} \alpha_{i} \tilde{x}_{i}\left(\left(\left[z_{3}\left(z_{0}\right)\right]+\left[z_{2 a}\left(z_{0}\right)\right]+\left[z_{2 b}\left(z_{0}\right)\right]+\left[z_{2 c}\left(z_{0}\right)\right]\right)\right. \\
- & \left.\left(\left[z_{3}^{\prime}\left(z_{0}\right)\right]+\left[z_{2 a}^{\prime}\left(z_{0}\right)\right]+\left[z_{2 b}^{\prime}\left(z_{0}\right)\right]+\left[z_{2 c}^{\prime}\left(z_{0}\right)\right]\right)\right) .
\end{aligned}
$$

Here, we note that

$\left(\left[z_{3}\left(z_{0}\right)\right]+\left[z_{2 a}\left(z_{0}\right)\right]+\left[z_{2 b}\left(z_{0}\right)\right]+\left[z_{2 c}\left(z_{0}\right)\right]\right)-\left(\left[z_{3}^{\prime}\left(z_{0}\right)\right]+\left[z_{2 a}^{\prime}\left(z_{0}\right)\right]+\left[z_{2 b}^{\prime}\left(z_{0}\right)\right]+\left[z_{2 c}^{\prime}\left(z_{0}\right)\right]\right) \in R_{00010}$.

Hence, by the assumption of Case $\epsilon_{4}=1$ and by Proposition 11, for each $z_{0}$,

$$
\begin{gathered}
\sum_{n_{b-1}+1 \leq i \leq n_{d}} \alpha_{i} \tilde{x}_{i}\left(\left(\left[z_{3}\left(z_{0}\right)\right]+\left[z_{2 a}\left(z_{0}\right)\right]+\left[z_{2 b}\left(z_{0}\right)\right]+\left[z_{2 c}\left(z_{0}\right)\right]\right)\right. \\
\left.\quad-\left(\left[z_{3}^{\prime}\left(z_{0}\right)\right]+\left[z_{2 a}^{\prime}\left(z_{0}\right)\right]+\left[z_{2 b}^{\prime}\left(z_{0}\right)\right]+\left[z_{2 c}^{\prime}\left(z_{0}\right)\right]\right)\right) \\
\quad=0 .
\end{gathered}
$$

These show that

$$
\sum_{n_{b-1}+1 \leq i \leq n_{d}} \alpha_{i} x_{i}(P)=\sum_{n_{b-1}+1 \leq i \leq n_{d}} \alpha_{i} x_{i}\left(P^{\prime}\right) .
$$

Hence, $\sum_{n_{b-1}+1 \leq i \leq n_{d}} \alpha_{i} x_{i}$ is invariant under strong RIII.

- (Proof of the case $\epsilon_{5}=1$.) Since the arguments are essentially the same as those for the case $\epsilon_{4}=1$, we omit this proof. 
4. Complete analysis of invariants obtained from $G_{2,4}$ And $R_{00010}(2,4)$

In this section, by using Corollary 2 , we completely analyze invariants obtained from $G_{2,4} \cap$ Conn and $R_{00010}(2,4)$. First, it is easy to see that $\left\{x_{i}\right\}_{i \in I_{2,4}^{(\mathrm{Conn})}}$ consists of nine elements, $y_{1}=\bigotimes, y_{2}=\bigotimes, y_{3}=\bigoplus, y_{4}=\bigoplus, y_{5}=\bigoplus, y_{6}=\bigotimes, y_{7}=$ $\otimes, y_{8}=\otimes$, and $y_{9}=\otimes$. This fact together with Corollary 2 implies that if there exist integers $\alpha_{i}(1 \leq i \leq 9)$ such that $\left(\sum_{i=1}^{9} \alpha_{i} \tilde{y}_{i}\right)(r)=0\left(\forall r \in R_{00010}(2,4)\right)$, then $\sum_{i=1}^{9} \alpha_{i} y_{i}$ is invariant under RI and strong RIII. Second, recall that $R_{00010}(2,4)$ consists of twelve elements $r_{j}(1 \leq j \leq 12)$ as follows (see Example 3):

$$
\begin{aligned}
& r_{1}=\otimes+3 \otimes-3 \mathbb{D}-\mathbb{Q} \text {, } \\
& r_{2}=\theta+3 \otimes-\theta-2 \mathbb{D}-\mathbb{O} \text {, } \\
& r_{3}=\bigotimes+\otimes+\bigoplus-\mathbb{Q}-2 \otimes \text {, } \\
& r_{4}=2 \otimes-\otimes-\otimes, \\
& r_{5}=\otimes+2 \bigoplus-\Theta-2 \bigotimes \text {, } \\
& r_{6}=\circledast+2 \otimes-\otimes-2 \otimes, \\
& r_{7}=2 \otimes+\otimes-2 \otimes-\otimes, \\
& r_{8}=\circledast+2 \otimes-\bigoplus-2 \mathbb{Q} \text {, } \\
& r_{9}=3-2 \geqslant-\theta \text {, } \\
& r_{10}=3 \partial-2 \ominus-\theta \text {, } \\
& r_{11}=3 \ominus-2 \ominus-0 \text {, and } \\
& r_{12}=\theta+2 \otimes-\theta-2 \theta \text {. }
\end{aligned}
$$

We can find $\alpha_{i}(1 \leq i \leq 9)$ satisfying $\left(\sum_{i=1}^{9} \alpha_{i} \tilde{y}_{i}\right)(r)=0\left(\forall r \in R_{00010}(2,4)\right)$

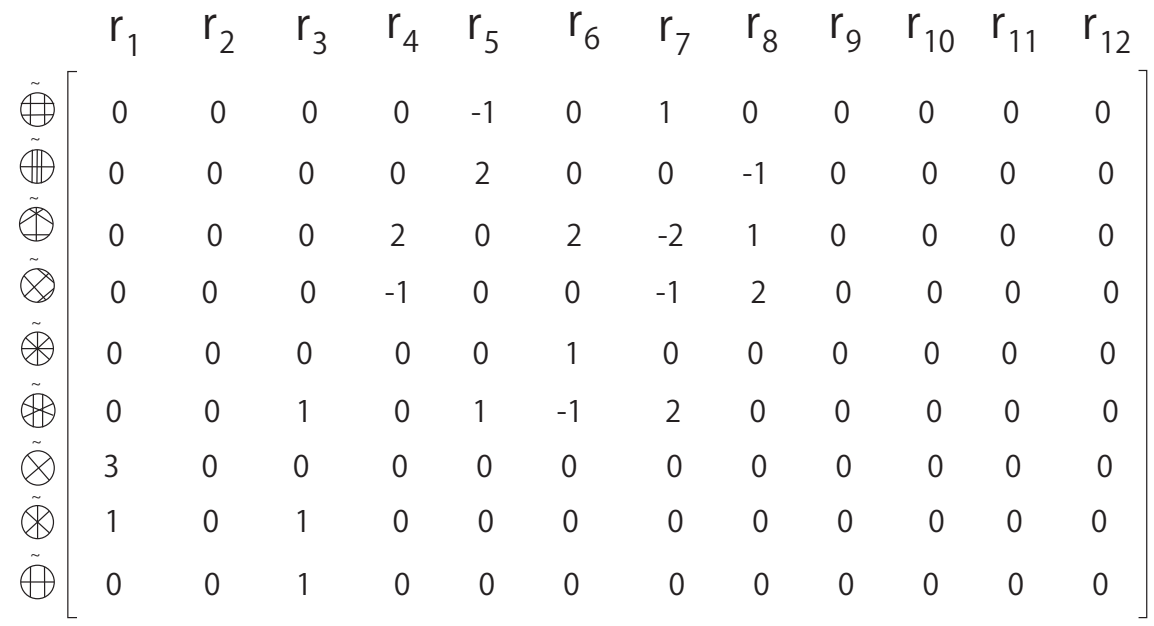

FiguRE 12. Matrix $\left(\tilde{y}_{i}\left(r_{j}\right)\right)_{1 \leq i \leq 9,1 \leq j \leq 12}$. 
by solving the linear equation $\mathbf{x} M=\mathbf{0}$, where $M=\left(\tilde{y}_{i}\left(r_{j}\right)\right.$ ) (Figure 12). It is elementary to show that the set of solutions is $\left\{\mathbf{x}=\gamma_{1}(1,-3,3,0,0,0,0,0,0)\right.$, $\left.\gamma_{2}(0,0,2,8,5,1,-4,2,-2) \mid \gamma_{1}, \gamma_{2} \in \mathbb{Z}\right\}$. Particularly, $y_{1}-3 y_{2}+3 y_{3}(=\otimes-3 \otimes$ $+3 \bigoplus)$ and $2 y_{3}+8 y_{4}+5 y_{5}+y_{6}-4 y_{7}+2 y_{8}-2 y_{9}(=2 \bigoplus+8 \bigoplus+5 \bigoplus+\circledast$ $-4 \circledast+2 \otimes-2 \circledast$ ) are invariant under RI and strong RIII.

Let $\lambda^{(3)}=y_{1}-3 y_{2}+3 y_{3}$ and let $\lambda^{(4)}=2 y_{3}+8 y_{4}+5 y_{5}+y_{6}-4 y_{7}+2 y_{8}-$ $2 y_{9}$. Note that $\lambda^{(3)}$ is the function obtained in Example 5 , hence $\lambda^{(3)}=4 \lambda$ where $\lambda$ is the function that appears in Theorem 1 of $[5]$. We further note that in [5], it is shown that $\lambda$ (hence, $\frac{1}{4} \lambda^{(3)}$ ) is a complete invariant of prime spherical curves up to seven double points (Table 1). We note that $\lambda^{(4)}$ is also a complete invariant of prime spherical curves up to seven double points. We further note that $\lambda^{(4)}$ is stronger than $\lambda^{(3)}$ in some sense. In fact, $\lambda\left(4_{1}\right)=\lambda\left(8_{9}\right)=4$ and $\lambda^{(4)}\left(4_{1}\right)(=16)$ $\neq \lambda^{(4)}\left(8_{9}\right)(=40)$. Similarly, we have $\lambda\left(5_{1}\right)=\lambda\left(8_{2}\right)=-5$ and $\lambda^{(4)}\left(5_{1}\right)(=-20)$ $\neq \lambda^{(4)}\left(8_{2}\right)(=-28)$, and $\lambda\left(8_{5}\right)=\lambda\left(8_{17}\right)=12$ and $\lambda^{(4)}\left(8_{5}\right)(=128) \neq \lambda^{(4)}\left(8_{17}\right)$ $(=116)$.

\section{ACKNOWLEDGEMENTS}

The author would like to thank Professors Tsuyoshi Kobayashi and Kouki Taniyama for helpful discussions. The author also would like to thank Mr. Yusuke Takimura for creating and providing many figures of spherical curves with indices (Table 1) and for useful discussions. Further, the author would like to thank Professor Yukari Funakoshi and Ms. Megumi Hashizume for their comments on an earlier version of this paper. The author would like to thank the referee for the comments. The author was a project researcher of Grant-in-Aid for Scientific Research (S) 24224002 (April 2016-March 2017).

\section{REFERENCES}

[1] R. Bott and C. Taubes, On the self-linking of knots, Topology and physics, J. Math. Phys. 35 (1994), 5247-5287.

[2] M. Goussarov, M. Polyak, and O. Viro, Finite-type invariants of classical and virtual knots, Topology 39 (2000), 1-45-1068.

[3] T. Hagge and J. Yazinski, On the necessity of Reidemeister move 2 for simplifying immersed planar curves, Banach Center Publ. 103 (2014), 101-110.

[4] N. Ito and Y. Takimura, $(1,2)$ and weak $(1,3)$ homotopies on knot projections, J. Knot Theory and Ramifications 22 (2013), 1350085, 14pp.

[5] N. Ito and Y. Takimura, Sub-chord diagrams of knot projections, Houston J. Math. 41 (2015), $701-725$

[6] N. Ito, Y. Takimura, and K. Taniyama, Strong and weak $(1,3)$ homotopies on knot projections, Osaka J. Math. $\mathbf{5 2}$ (2015), 617-646.

[7] O.-P. Östlund, A diagrammatic approach to link invariants of finite degree, Math. Scand. 94 (2004), 295-319.

[8] M. Polyak and O. Viro, Gauss diagram formulas for Vassiliev invariants, Internat. Math. Res. Notices 1994, 445ff., approx. 8pp. (electronic).

[9] V. A. Vassiliev, Cohomology of knot spaces, Theory of singularities and its applications, 23-69, Adv. Soviet Math., 1, Amer. Math. Soc., Providence, RI, 1990.

[10] O. Viro, Generic immersions of the circle to surfaces and the complex topology of real algebraic curves. Topology of real algebraic varieties and related topics, 231-252, Amer. Math. Soc. Transl. Ser. 2, 173, Adv. Math. Sci., 29, Amer. Math. Soc., Providence, RI, 1996.

Graduate School of Mathematical Sciences, The University of Tokyo, 3-8-1, Komaba, Meguro-Ku, TOKYO 153-8914, JAPAN

Email address: noboru@ms.u-tokyo.ac.jp 
TABLE 1. Values of $\frac{1}{4} \lambda^{(3)}(=\lambda)$ and $\lambda^{(4)}$ for prime spherical curves with at most seven double points. Any pair of prime spherical curves in the same box in the leftmost column are related by a finite sequence of Reidemeister moves of types RI and strong RIII.

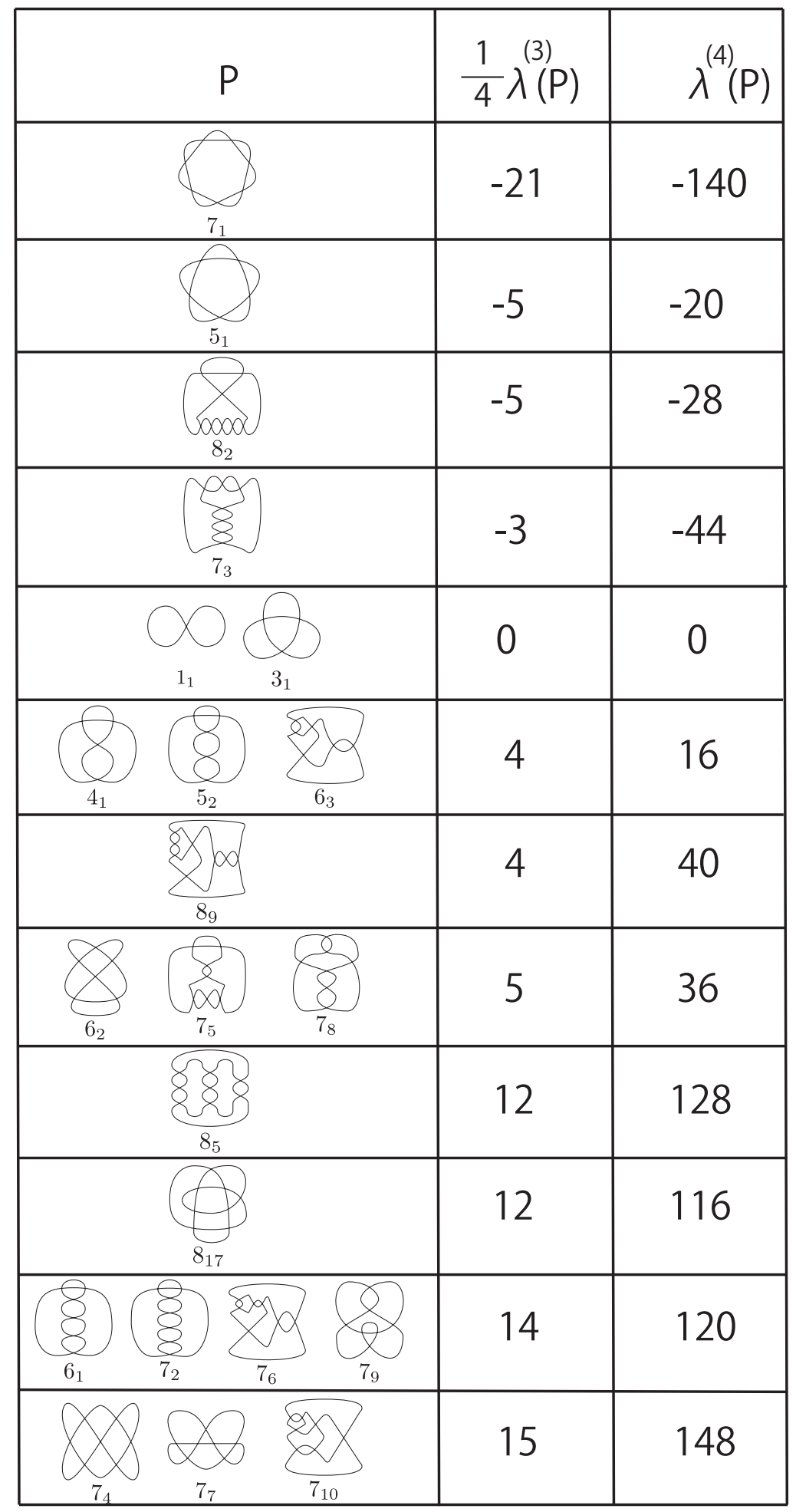

